\title{
Theoretical Calculations, Microwave Spectroscopy, and Ring-Puckering Vibrations of 1,1-Dihalosilacyclopent-2-enes
}

Thomas M. C. McFadden ${ }^{a}$, Frank E. Marshall ${ }^{b}$, Esther J. Ocola ${ }^{c}$, Jaan Laane ${ }^{c^{*}}$, Gamil A. Guirgis $^{a}$, and Garry S. Grubbs II ${ }^{b^{*}}$

${ }^{a}$ College of Charleston, Department of Chemistry and Biochemistry, 66 George St., Charleston, SC, USA, 29424

${ }^{\mathrm{b}}$ Missouri University of Science and Technology, Department of Chemistry, 400 W. 11th St., Rolla, MO, USA, 65409

${ }^{\mathrm{c}}$ Texas A\&M University, Department of Chemistry and Institute for Quantum Science and Engineering, College Station, TX, USA, 77843

\section{SUPPORTING INFORMATION}

S1-S7 are the microwave transition assignments and fits for all reported isotopologues of 1,1Difluorosilacyclopent-2-ene. Transition assignments are given in $J K_{a} K_{c}{ }^{\prime} \leftarrow J K_{a} K_{c}$ "' The experimental frequencies are given in MHz.

\section{Table of Contents}

S1. Parent MW Transition Assignments

Page

S2. $\quad{ }^{29}$ Si MW Transition Assignments

S3. $\quad{ }^{30}$ Si MW Transition Assignments

S4. $\quad{ }^{13} \mathrm{C}_{1}$ MW Transition Assignments

S5. ${ }^{13} \mathrm{C}_{2}$ MW Transition Assignments

S6. $\quad{ }^{13} \mathrm{C}_{3}$ MW Transition Assignments

S22-S25

S7. ${ }^{13} \mathrm{C}_{4} \mathrm{MW}$ Transition Assignments

S8. Output of Structure Evaluation Program for Ring Bond Lengths, Angles, and Dihedral Angles (units are given in $\AA$, degrees, and degrees, respectively) in 1,1Difluorosilacyclopent-2-ene

S9. Output of Kraitchman Analysis Program (atom positions are absolute and given in $\AA$ ) in 1,1-Difluorosilacyclopent-2-ene

\footnotetext{
*Corresponding authors: Jaan Laane and Garry S. Grubbs II.
} 


\section{S1. Parent MW Transition Assignments}

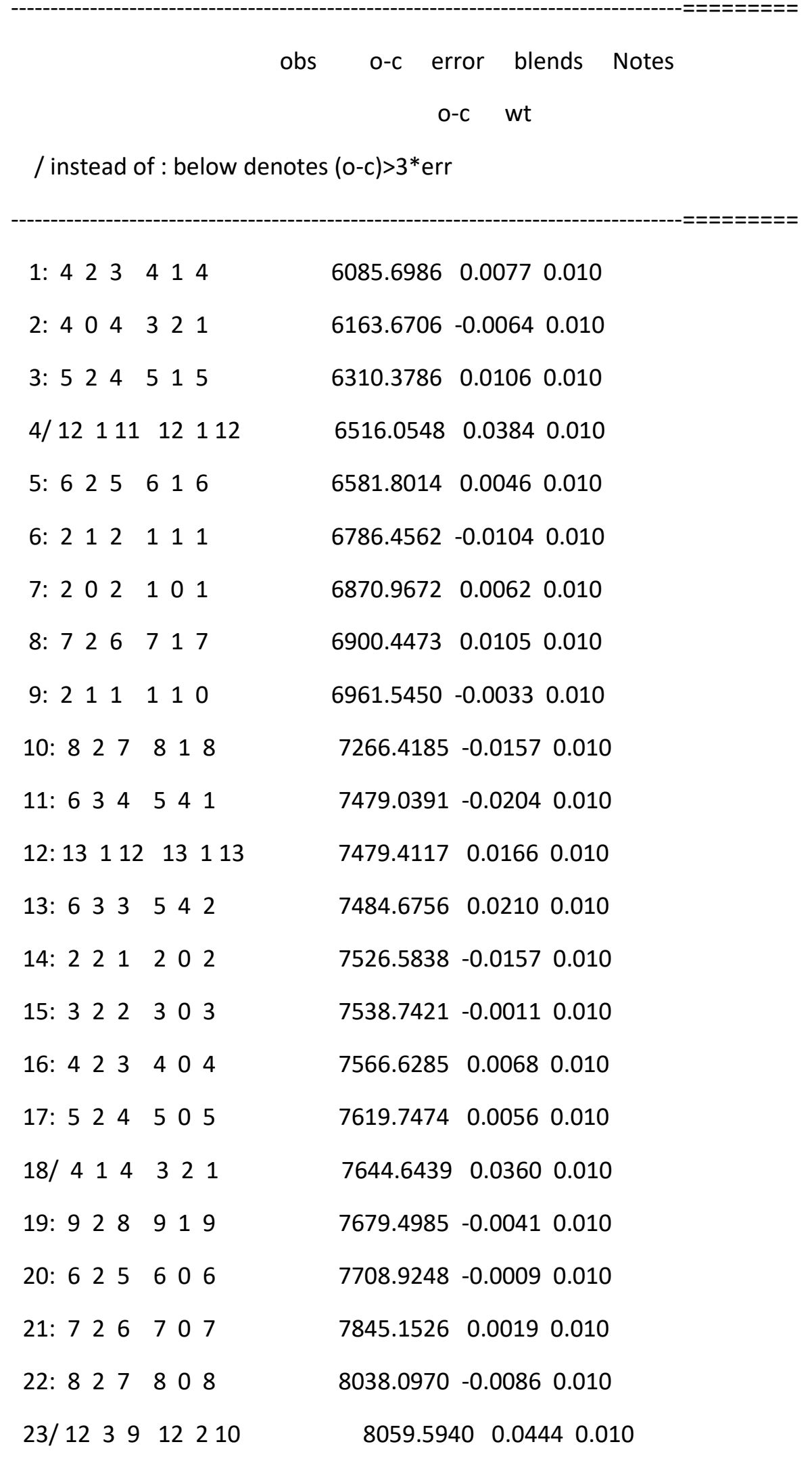


$24 / 102910110$

25: 928909

26: 11381129

27: $4133 \quad 322$

28: $303 \quad 212$

29: 10371028

30: 102910010

31: 212101

32: $936 \quad 927$

33: 835826

$34 / 1121011011$

35: 734725

36: $6333 \quad 624$

37: 532523

38: 431422

39: 3300321

40/ 331322

41: 4324423

42: $5 \begin{array}{lllll}3 & 3 & 5 & 2 & 4\end{array}$

43: 1511415115

44: 634625

45: 735726

46: $505 \quad 422$

47: $83 \quad 6 \quad 8227$

48: $937 \quad 928$

49: 10381029

50: 113911210

51: 3132212

52: 303202
$8138.7967-0.03040 .010$

$8294.8321-0.01370 .010$

$8329.3831-0.01320 .010$

$8534.8096 \quad 0.0040 \quad 0.010$

$\begin{array}{lll}8546.1974 & 0.0038 & 0.010\end{array}$

$8585.1156 \quad 0.0030 \quad 0.010$

$8618.9631-0.01820 .010$

8623.59020 .00320 .010

$8812.5545 \quad 0.02330 .010$

$9002.1813-0.00180 .010$

$9010.5851-0.03290 .010$

$9149.8995-0.00270 .010$

$9256.6167-0.00040 .010$

$\begin{array}{lll}9327.3180 & 0.01090 .010\end{array}$

$\begin{array}{lll}9369.4135 & 0.0088 & 0.010\end{array}$

$\begin{array}{lll}9391.1225 & 0.00030 .010\end{array}$

9406.34450 .03550 .010

9414.52690 .00170 .010

$\begin{array}{lll}9431.0501 & 0.0107 & 0.010\end{array}$

$9455.5960-0.01590 .010$

9459.67090 .01130 .010

$9504.6004-0.01290 .010$

$9512.7524-0.00680 .010$

$9570.3694-0.01330 .010$

$9661.5106-0.01870 .010$

$9782.5062-0.01610 .010$

$9937.5463-0.02810 .010$

$10177.8059-0.00670 .010$

$\begin{array}{lll}10298.8245 & 0.0051 & 0.010\end{array}$ 


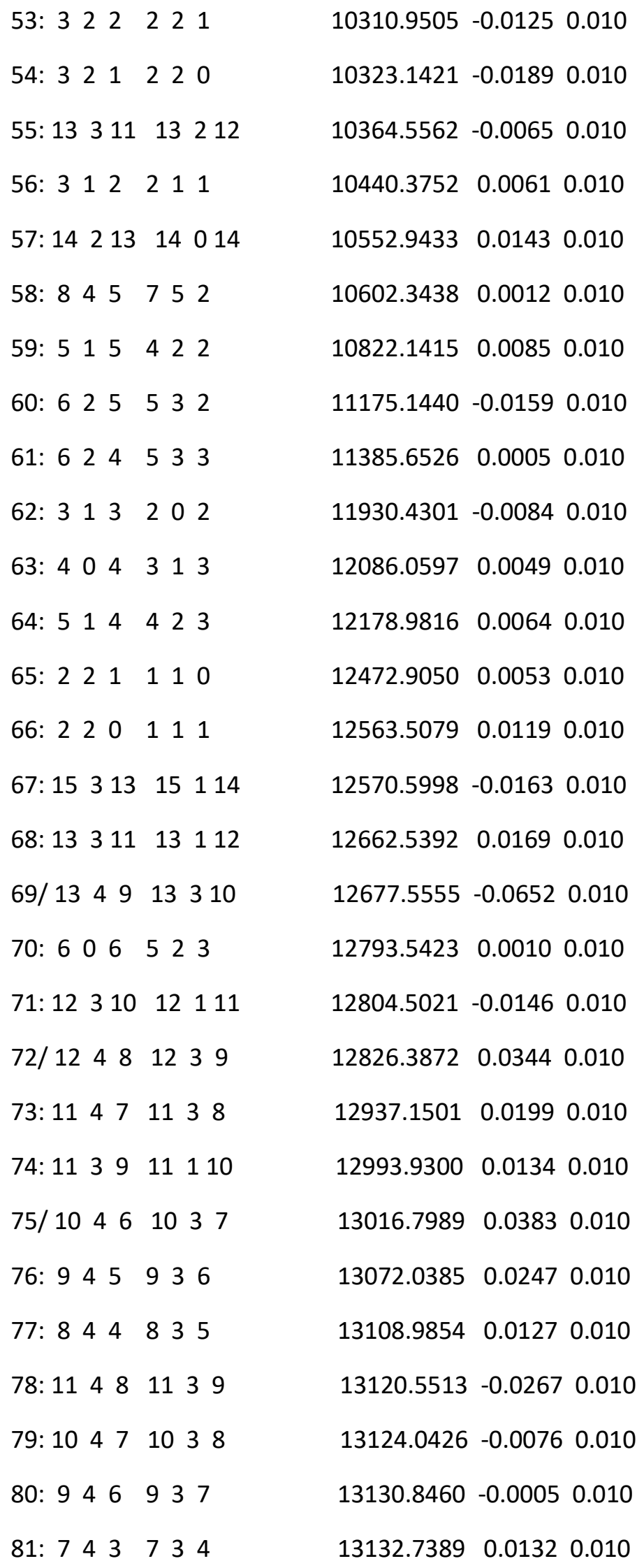


$82 / 13410 \quad 13311$

83: $845 \quad 836$

84: 744735

85: 642633

86: $643 \quad 634$

87/ 541532

88: 542533

89: 10381019

90: 937918

91: $414 \quad 313$

92: $404 \quad 303$

93: $423 \quad 322$

94: 432331

95: 4313330

96: 422321

97: 8177334

98: 413312

99: 616523

100: 735716

101: 634615

102: 836743

103/ 220101

104/ 5333514

105: 726633

106: 432413

107: 414303

108/431 414

109: $505 \quad 414$

110: 322211 $\begin{array}{lll}13135.5736 & 0.0305 & 0.010\end{array}$

$\begin{array}{lll}13138.8268 & 0.0186 & 0.010\end{array}$

$\begin{array}{lll}13146.4460 & 0.0101 & 0.010\end{array}$

$\begin{array}{lll}13147.3212 & 0.0173 \quad 0.010\end{array}$

$13152.8480 \quad 0.0144 \quad 0.010$

$\begin{array}{lll}13155.8018 & 0.0419 & 0.010\end{array}$

$13157.6035-0.01110 .010$

$\begin{array}{lll}13217.3995 & 0.0103 \quad 0.010\end{array}$

$13461.9839-0.0096 \quad 0.010$

$13566.9805-0.0050 \quad 0.010$

$\begin{array}{lll}13717.6828 & 0.0089 & 0.010\end{array}$

$13745.5400-0.01230 .010$

$13753.7595-0.00900 .010$

$13754.1627-0.00610 .010$

$13775.8712-0.0150 \quad 0.010$

$\begin{array}{lll}13794.5074 & 0.0024 & 0.010\end{array}$

$13916.7605 \quad 0.00960 .010$

$13920.6540-0.01610 .010$

$13967.7963 \quad 0.01240 .010$

$14208.6481-0.0178 \quad 0.010$

14377.52290 .01880 .010

$14400.65490 .0386 \quad 0.010$

$\begin{array}{lll}14430.1219 & -0.0308 & 0.010\end{array}$

14569.75420 .01260 .010

$14625.2616-0.0103 \quad 0.010$

$15198.6034-0.0013 \quad 0.010$

$15500.6504-0.03260 .010$

15644.06290 .02530 .010

$15822.3030-0.01120 .010$ 


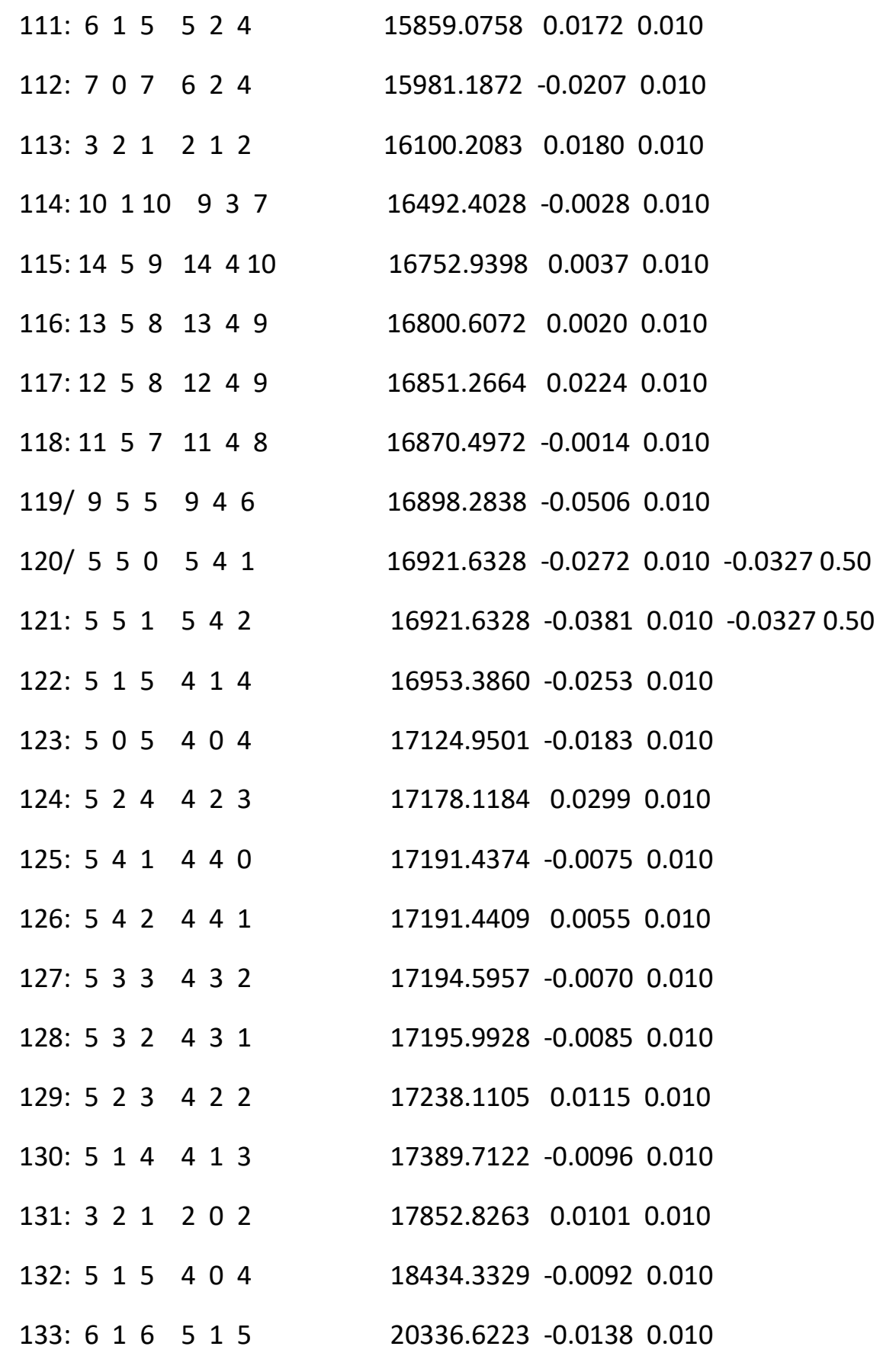

PARAMETERS IN FIT (values truncated):

$\begin{array}{llll}10000 & \mathrm{~A} / \mathrm{MHz} & 3599.3985(6) & 1 \\ 20000 & \mathrm{~B} / \mathrm{MHz} & 1762.2756(3) & 2 \\ 30000 & \mathrm{C} / \mathrm{MHz} & 1674.7349(3) & 3\end{array}$




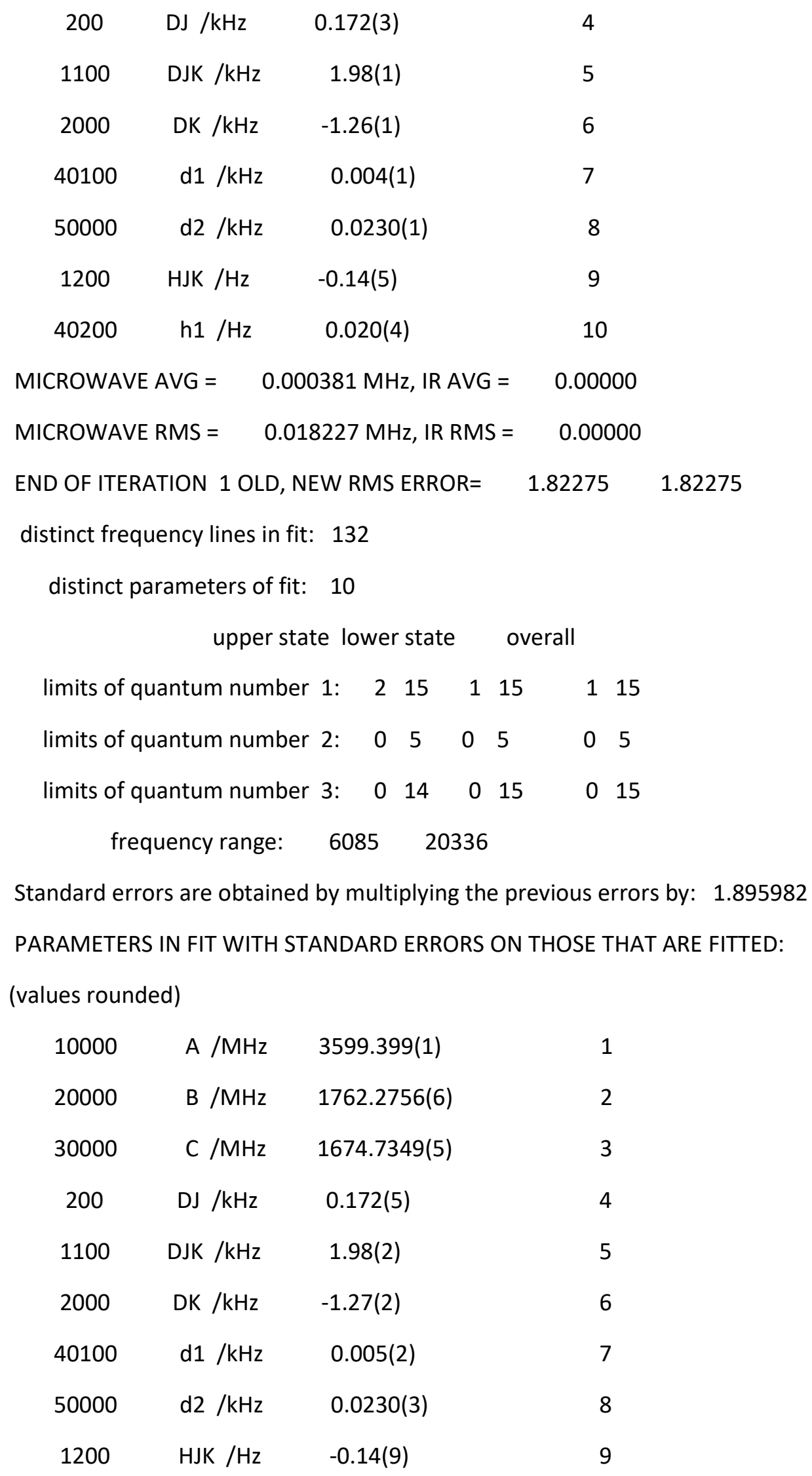


$\begin{array}{llll}40200 & \mathrm{~h} 1 / \mathrm{Hz} & 0.020(7) & 10\end{array}$

\begin{tabular}{ccccc}
\multicolumn{5}{c}{ Worst fitted constants, with greater than $20 \%$ uncertainty: } \\
$\begin{array}{ccccc}40100 & \mathrm{~d} 1 / \mathrm{kHz} & 0.005(2) & 7 & 46.3 \\
1200 & \mathrm{HJK} / \mathrm{Hz} & -0.14(9) & 9 & 67.1 \\
40200 & \mathrm{~h} 1 / \mathrm{Hz} & 0.020(7) & 10 & 37.7\end{array}$
\end{tabular}

CORRELATION COEFFICIENTS, C.ij:

$\begin{array}{lllllllll}A & B & C & -D J & -D J K & -D K & d 1 & d 2\end{array}$
A $\quad 1.0000$
B $\quad 0.4622 \quad 1.0000$
C $\quad 0.4555 \quad 0.3298 \quad 1.0000$
-DJ $\quad-0.3385-0.6400-0.6963 \quad 1.0000$

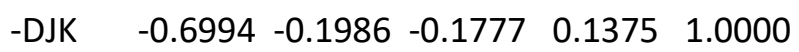

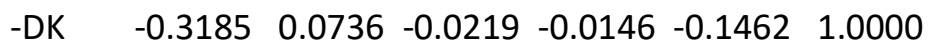

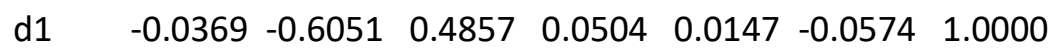

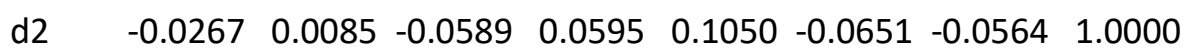
$\begin{array}{lllllllll}\text { HJK } & 0.6305 & 0.1730 & 0.1763 & -0.1604 & -0.9579 & 0.0601 & 0.0069 & -0.0786\end{array}$
h1 $\quad 0.0272 \quad 0.5578 \quad-0.4324 \quad-0.0678 \quad 0.0150 \quad 0.0374 \quad-0.9695 \quad 0.1922$

HJK h1

HJK 1.0000

h1 $-0.0336 \quad 1.0000$

Mean value of $\mid$ C.ij $\mid$, i.ne.j $=0.2426$

Mean value of C.ij, i.ne.j $=-0.0622$

No correlations with absolute value greater than 0.9950

Worst fitted lines (obs-calc/error):

69: $-6.5 \quad 119:-5.1 \quad 23: 4.4 \quad 87: 4.2$

103: $3.9 \quad 4: \quad 3.8 \quad 75: \quad 3.8 \quad 18: 3.6$

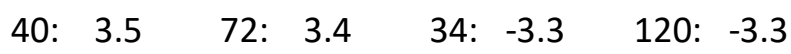




\begin{tabular}{|c|c|c|c|c|c|c|c|}
\hline 108: & -3.3 & 104: & -3.1 & & : 3.0 & 24: $\quad-3.0$ & \\
\hline 124: & 3.0 & 50: & -2.8 & 78: & -2.7 & 122: -2.5 & \\
\hline 109: & 2.5 & 76: & 2.5 & $32:$ & 2.3 & 117: 2.2 & \\
\hline 13: & 2.1 & 112: & -2.1 & 11: & -2.0 & 73: $\quad 2.0$ & \\
\hline 54: & -1.9 & 102: & 1.9 & 48: & -1.9 & 83: 1.9 & \\
\hline 123: & -1.8 & 30: & -1.8 & 113: & : 1.8 & 101: -1.8 & \\
\hline 85: & 1.7 & 111: & 1.7 & 68: & 1.7 & 12: 1.7 & \\
\hline 67: & -1.6 & 49: & -1.6 & 99: & -1.6 & 43: -1.6 & \\
\hline 60: & -1.6 & 14: & -1.6 & 10: & -1.6 & 96: -1.5 & \\
\hline 71: & -1.5 & 86: & 1.4 & & & & \\
\hline $69 /$ & 1349 & 133 & & & 12677.5 & $555-0.0652$ & 20.010 \\
\hline $119 /$ & 955 & 946 & & & L6898.28 & $838-0.0506$ & 0.010 \\
\hline $23 /$ & 1239 & 122 & & & $8059.5 \mathrm{~s}$ & $940 \quad 0.0444$ & 0.010 \\
\hline $87 /$ & 541 & 532 & & & 13155.8 & $018 \quad 0.0419$ & 90.010 \\
\hline $103 /$ & 220 & 101 & & & 14400.65 & $549 \quad 0.0386$ & 0.010 \\
\hline $4 / 1$ & 12111 & 121 & & & 6516.05 & $548 \quad 0.0384$ & 0.010 \\
\hline $75 /$ & 1046 & 103 & & & 13016.79 & 9890.0383 & 0.010 \\
\hline $18 /$ & 414 & 321 & & & 7644.64 & 4390.0360 & 0.010 \\
\hline $40 /$ & 331 & 322 & & & 9406.34 & $445 \quad 0.0355$ & 0.010 \\
\hline $72 /$ & 1248 & 123 & & & 12826.3 & $3872 \quad 0.0344$ & 40.010 \\
\hline
\end{tabular}

/ SPFIT output reformatted with PIFORM 


\section{S2. ${ }^{29} \mathrm{Si}$ MW Transition Assignments}

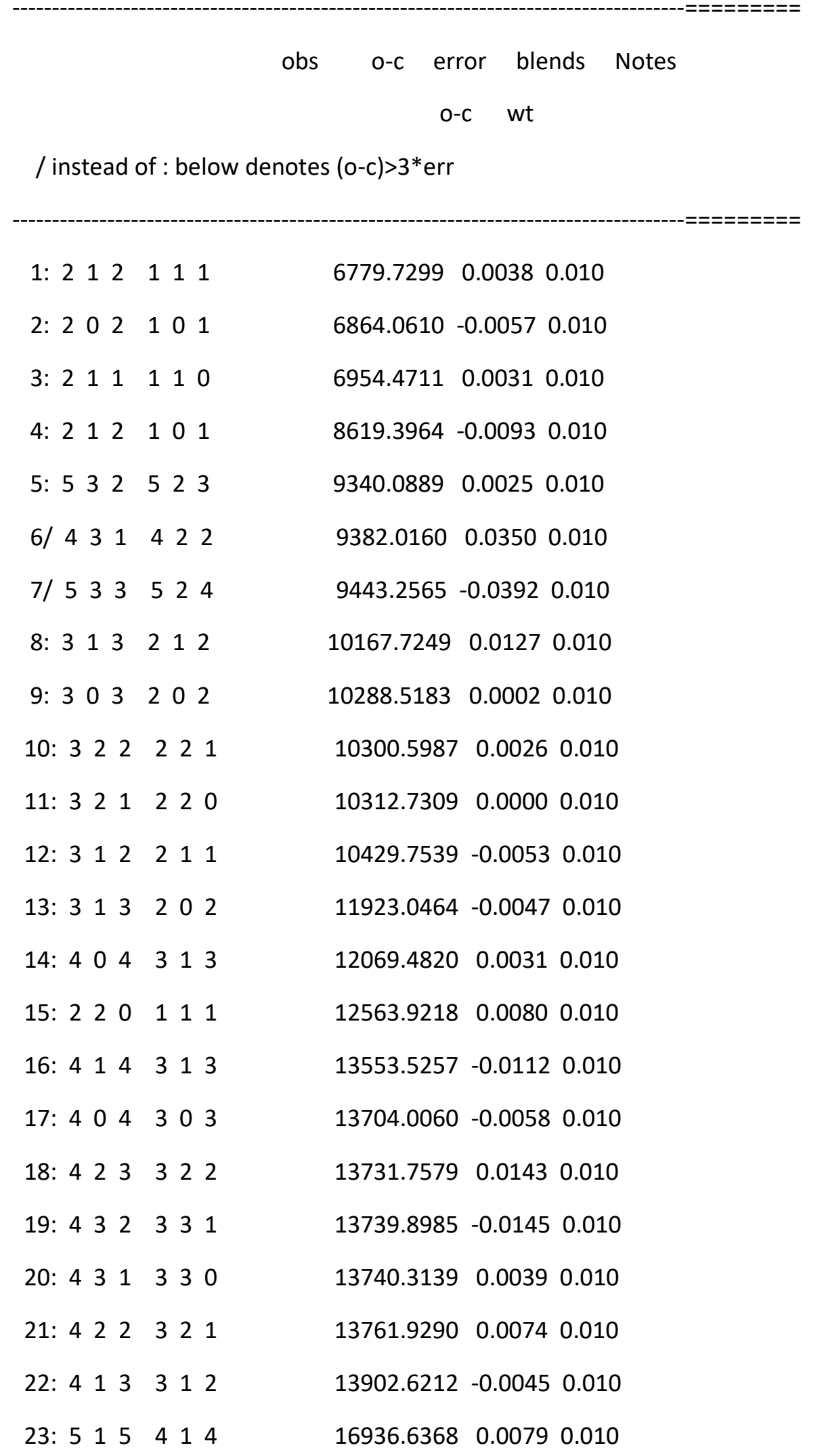




$\begin{array}{lllllllllll}\text { 24: } 5 & 0 & 5 & 4 & 0 & 4 & 17107.9986 & -0.0040 & 0.010 & \\ \text { 25: } & 5 & 2 & 4 & 4 & 2 & 3 & 17160.8272 & -0.0223 & 0.010 & \\ \text { 26/ } 5 & 4 & 2 & 4 & 4 & 1 & 17174.1494 & 0.0361 & 0.010 & 0.0313 & 0.50 \\ \text { 27: } & 5 & 4 & 1 & 4 & 4 & 0 & 17174.1494 & 0.0265 & 0.010 & 0.03130 .50 \\ \text { 28: } & 5 & 3 & 3 & 4 & 3 & 2 & 17177.2874 & 0.0128 & 0.010 & \\ \text { 29/ } 5 & 3 & 2 & 4 & 3 & 1 & 17178.6272 & -0.0342 & 0.010 & \\ \text { 30: } 5 & 2 & 3 & 4 & 2 & 2 & 17220.5429 & -0.0130 & 0.010 \\ \text { 31: } 5 & 1 & 4 & 4 & 1 & 3 & 17372.1255 & 0.0240 & 0.010\end{array}$

PARAMETERS IN FIT (values truncated):

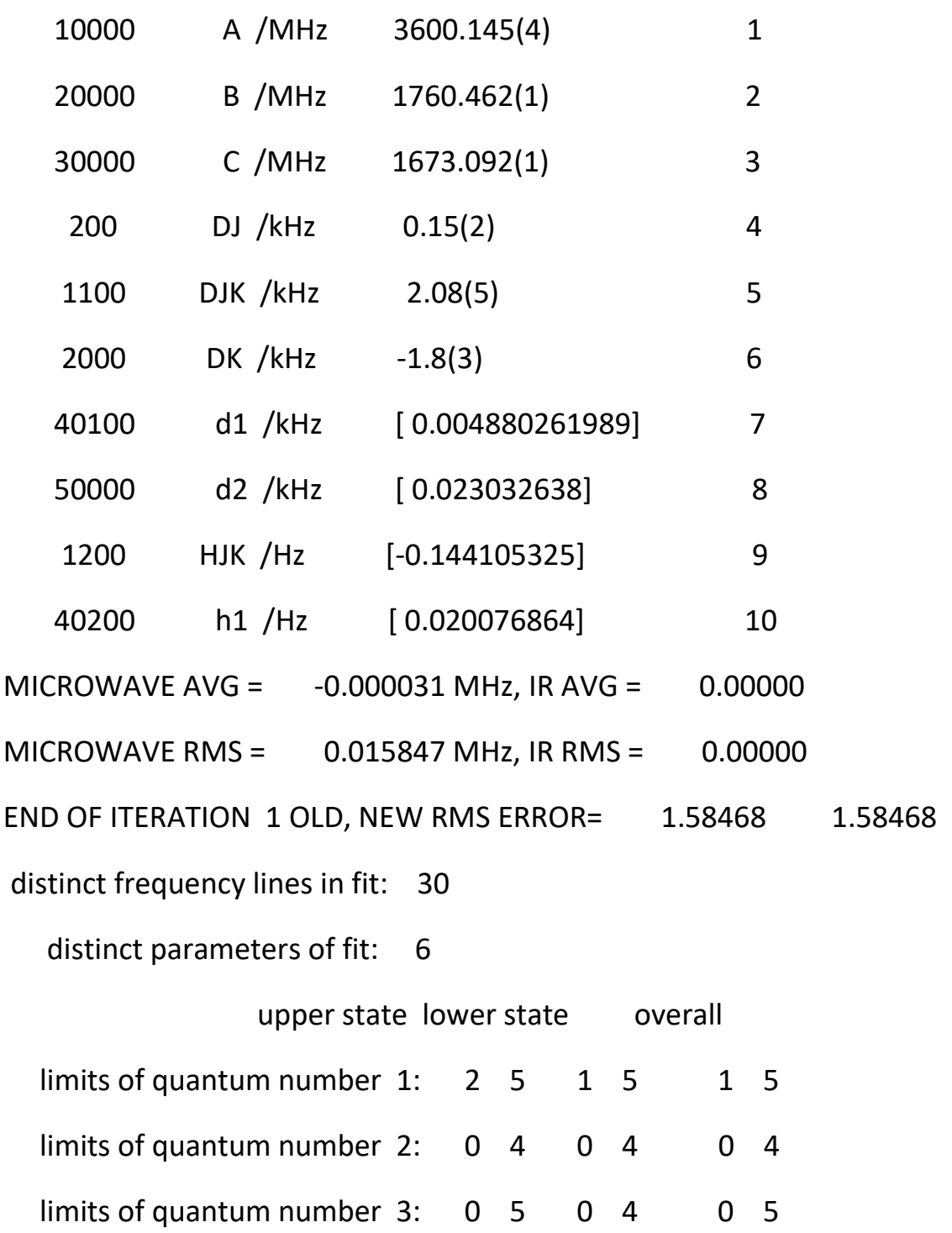


frequency range: $6779 \quad 17372$

Standard errors are obtained by multiplying the previous errors by: 1.771726

PARAMETERS IN FIT WITH STANDARD ERRORS ON THOSE THAT ARE FITTED:

(values rounded)

$\begin{array}{ccll}10000 & \mathrm{~A} / \mathrm{MHz} & 3600.145(7) & 1 \\ 20000 & \mathrm{~B} / \mathrm{MHz} & 1760.463(1) & 2 \\ 30000 & \mathrm{C} / \mathrm{MHz} & 1673.092(1) & 3 \\ 200 & \mathrm{DJ} / \mathrm{kHz} & 0.16(3) & 4 \\ 1100 & \mathrm{DJK} / \mathrm{kHz} & 2.1(1) & 5 \\ 2000 & \mathrm{DK} / \mathrm{kHz} & -1.8(6) & 6 \\ 40100 & \mathrm{~d} 1 / \mathrm{kHz} & {[0.004880261989]} & 7 \\ 50000 & \mathrm{~d} 2 / \mathrm{kHz} & {[0.023032638]} & 8 \\ 1200 & \mathrm{HJK} / \mathrm{Hz} & {[-0.144105325]} & 9 \\ 40200 & \mathrm{~h} 1 / \mathrm{Hz} & {[0.020076864]} & 10\end{array}$

Worst fitted constants, with greater than $20 \%$ uncertainty:

$\begin{array}{lllll}200 & \text { DJ } / \mathrm{kHz} & 0.16(3) & 4 & 22.9 \\ 2000 & \mathrm{DK} / \mathrm{kHz} & -1.8(6) & 6 & 36.6\end{array}$

CORRELATION COEFFICIENTS, C.ij:

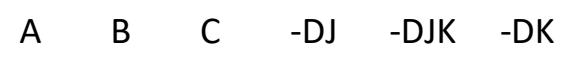

A $\quad 1.0000$

B $\quad 0.0914 \quad 1.0000$

C $\quad-0.2895 \quad 0.0570 \quad 1.0000$

-DJ $\quad 0.1397 \quad-0.6407 \quad-0.6738 \quad 1.0000$

$\begin{array}{llllll}\text {-DJK } & -0.0484 & -0.0276 & 0.0853 & -0.3398 & 1.0000\end{array}$

$\begin{array}{lllllll}\text {-DK } & -0.9083 & 0.0645 & 0.3255 & -0.1620 & -0.2646 & 1.0000\end{array}$

Mean value of $\mid$ C.ij $\mid$, i.ne.j $=0.2745$

Mean value of C.ij, i.ne.j $=-0.1728$ 
No correlations with absolute value greater than 0.9950

Worst fitted lines (obs-calc/error):

$\begin{array}{llllllll}\text { 7: } & -3.9 & 6: & 3.5 & 29: & -3.4 & 26: & 3.1 \\ \text { 31: } & 2.4 & 25: & -2.2 & 19: & -1.4 & 18: & 1.4 \\ \text { 30: } & -1.3 & 28: & 1.3 & 8: & 1.3 & 16: & -1.1 \\ \text { 4: } & -0.9 & 15: & 0.8 & 23: & 0.8 & 21: & 0.7 \\ \text { 17: } & -0.6 & 2: & -0.6 & 12: & -0.5 & 13: & -0.5 \\ \text { 22: } & -0.4 & 24: & -0.4 & 20: & 0.4 & 1: & 0.4 \\ \text { 3: } & 0.3 & 14: & 0.3 & 10: & 0.3 & 5: & 0.2 \\ \text { 9: } & 0.0 & 27: & 0.0 & 11: & 0.0 & & \end{array}$

7/ $5335524 \quad 9443.2565-0.03920 .010$

6/ $4314422 \quad 9382.01600 .03500 .010$

29/ 532443110.0178

26/ $5424441 \quad 17174.14940 .03610 .010 \quad 0.03130 .50$

31: $514413 \quad 17372.12550 .02400 .010$

25: $524423 \quad 17160.8272-0.02230 .010$

19: $432 \begin{array}{lllllll}3 & 3 & 1 & 13739.8985 & -0.0145 & 0.010\end{array}$

18: $423322 \quad 13731.75790 .01430 .010$

30: $523 \quad 422 \quad 17220.5429-0.01300 .010$

28: $\begin{array}{llllllll}3 & 3 & 4 & 3 & 2 & 17177.2874 & 0.0128 & 0.010\end{array}$

/ SPFIT output reformatted with PIFORM 


\section{S3. ${ }^{30}$ Si MW Transition Assignments}

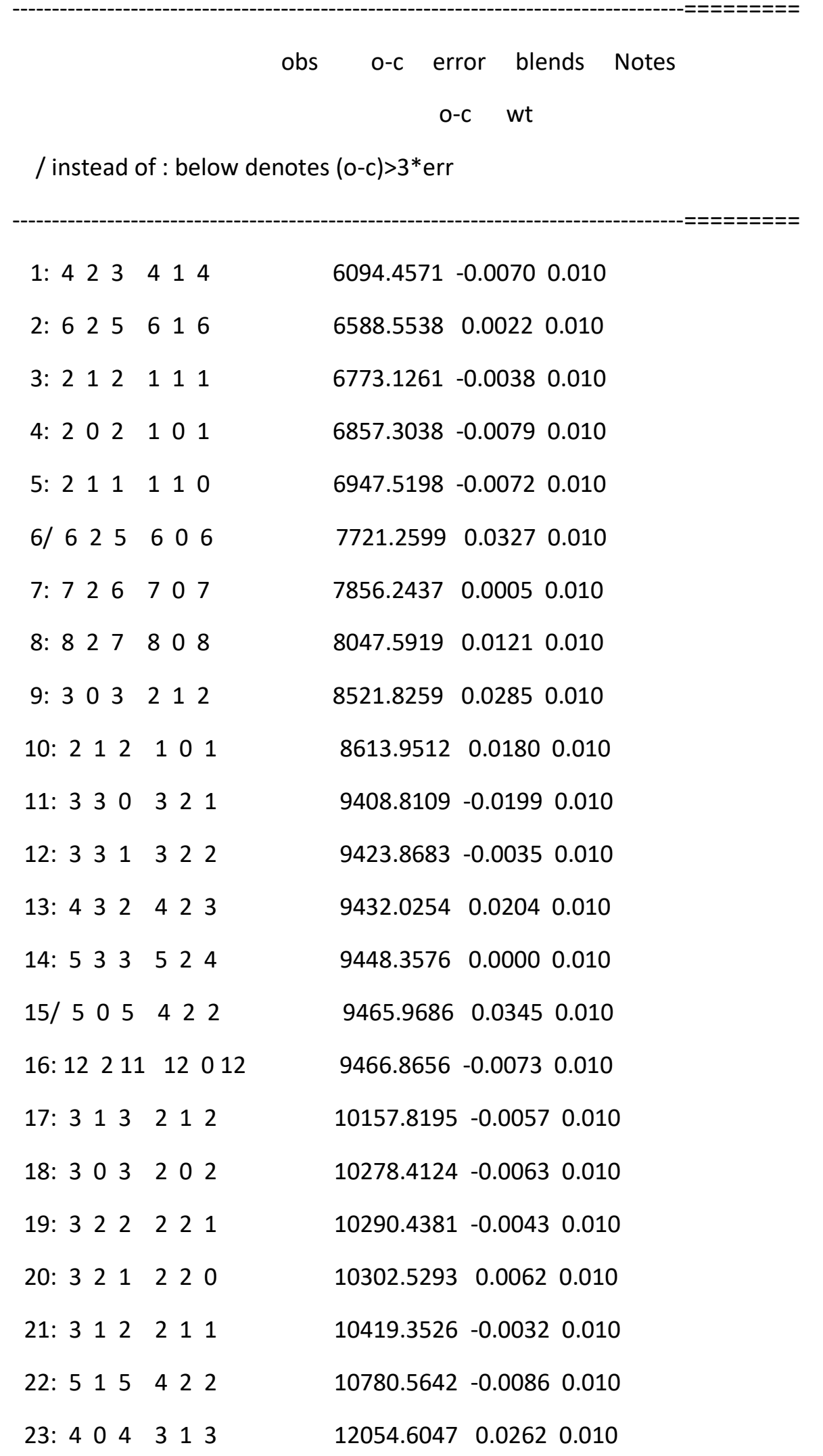




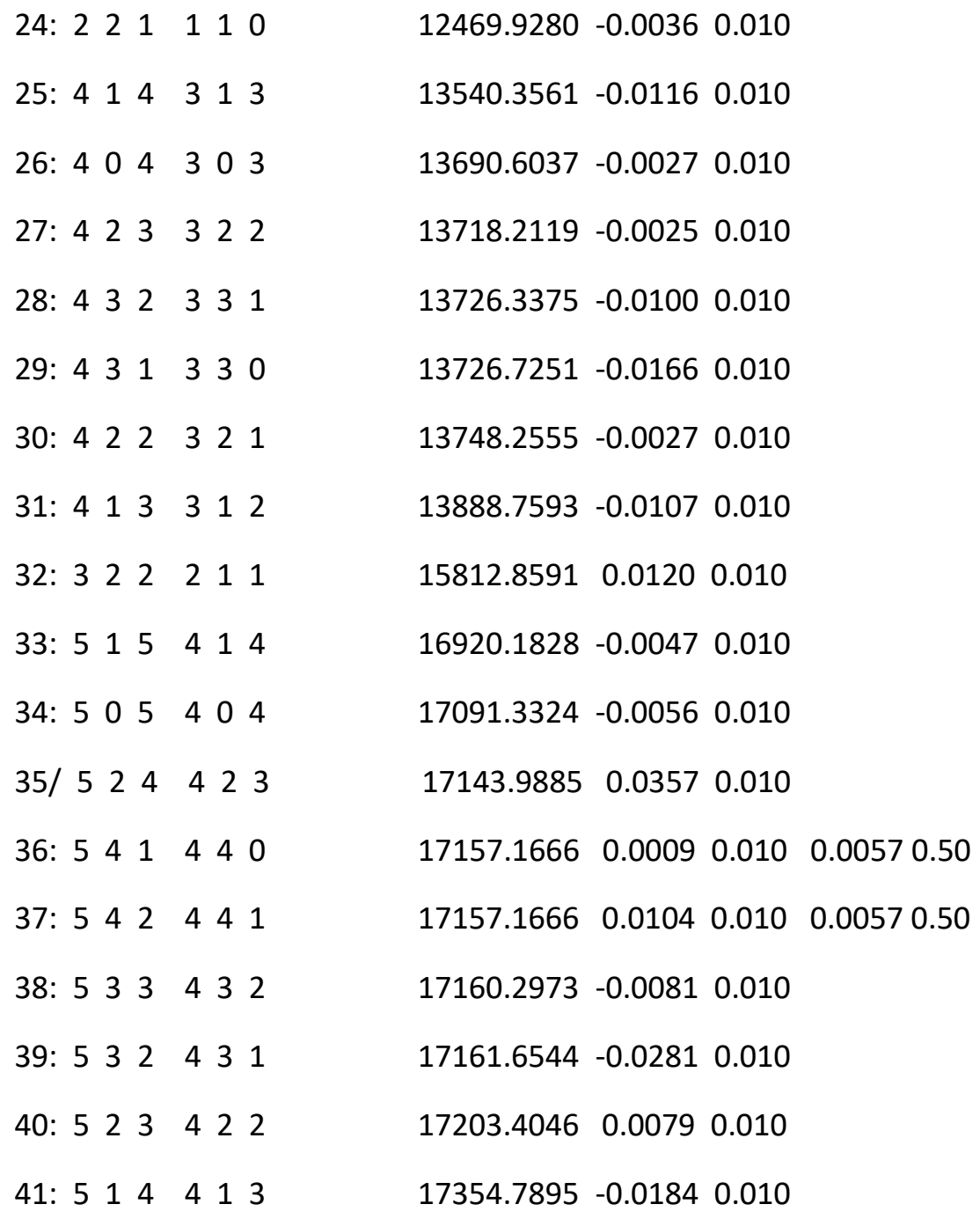

PARAMETERS IN FIT (values truncated):

$\begin{array}{ccll}10000 & \mathrm{~A} / \mathrm{MHz} & 3599.490(2) & 1 \\ 20000 & \mathrm{~B} / \mathrm{MHz} & 1758.6848(7) & 2 \\ 30000 & \mathrm{C} / \mathrm{MHz} & 1671.4863(8) & 3 \\ 200 & \mathrm{DJ} / \mathrm{kHz} & 0.17(1) & 4 \\ 1100 & \mathrm{DJK} / \mathrm{kHz} & 2.08(4) & 5 \\ 2000 & \mathrm{DK} / \mathrm{kHz} & -1.6(1) & 6 \\ 40100 & \mathrm{~d} 1 / \mathrm{kHz} & {[0.004880261989]} & 7 \\ 50000 & \mathrm{~d} 2 / \mathrm{kHz} & {[0.023032638]} & 8 \\ 1200 & \mathrm{HJK} / \mathrm{Hz} & {[-0.144105325]} & 9\end{array}$




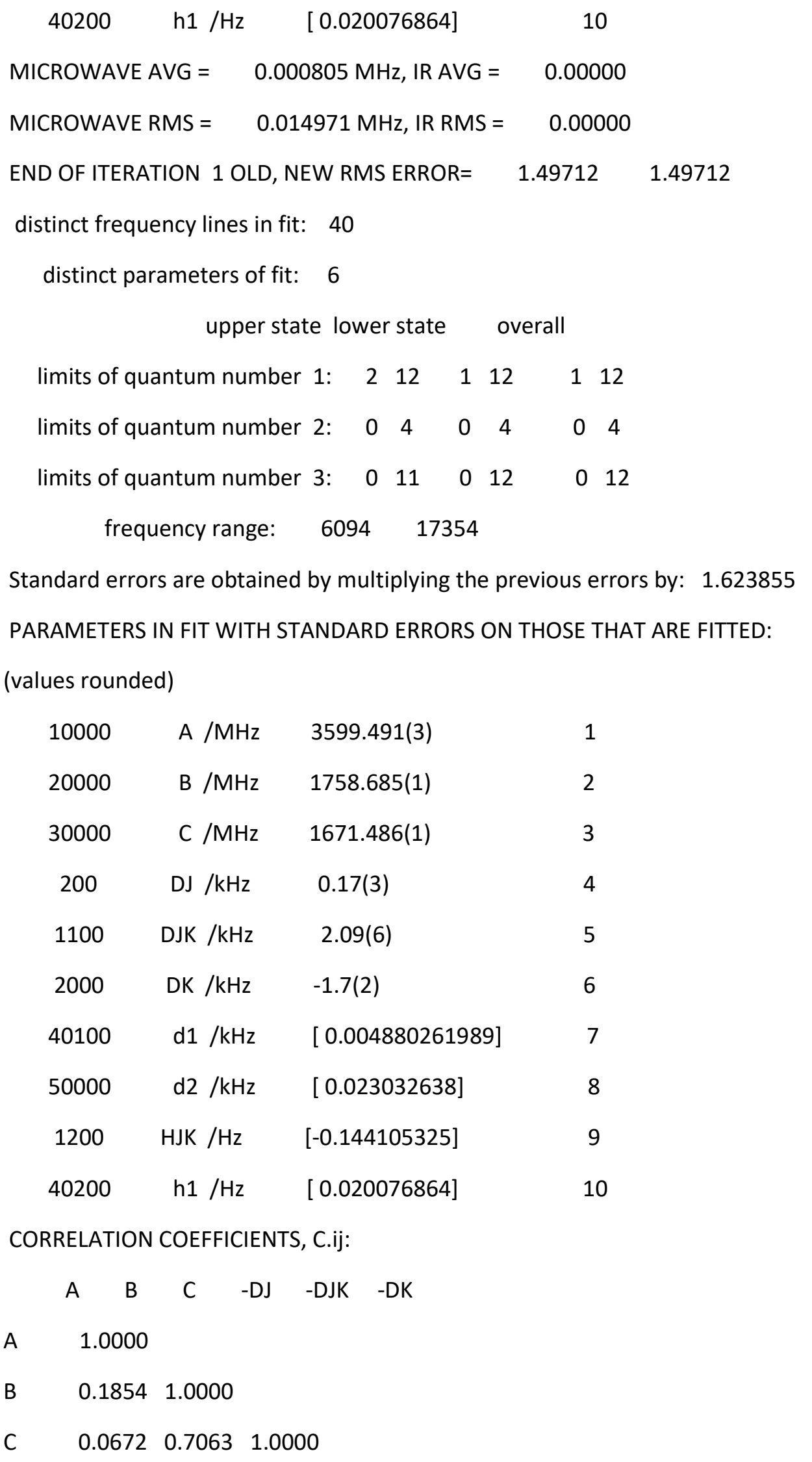




\begin{tabular}{|c|c|c|c|c|}
\hline -DJ & 0.0403 & $3-0.7599-($ & $\begin{array}{ll}-0.9318 & 1.0000\end{array}$ & \\
\hline -DJK & -0.4516 & $6-0.2382$ & $0.4204-0.3534$ & 1.0000 \\
\hline -DK & -0.8083 & 30.1847 & $0.0936-0.2054 \quad 0$ & 0.12351 .0000 \\
\hline Mear & value of & f $\mid$ C.ij|, i.ne & $e . j=0.3713$ & \\
\hline Mear & value of & f C.ij, i.ne.j & $j=-0.1285$ & \\
\hline No co & rrelation & Is with abso & solute value greater & r than 0.9950 \\
\hline Wors & t fitted lin & nes (obs-cal & alc/error): & \\
\hline 35: & 3.6 & 15: 3.5 & 6: 3.3 & 9: 2.9 \\
\hline 39: & -2.8 & 23: 2.6 & 13: 2.0 & 11: -2.0 \\
\hline 41: & -1.8 & 10: 1.8 & 29: -1.7 & 8: 1.2 \\
\hline 32: & 1.2 & $25:-1.2$ & 31: -1.1 & 28: -1.0 \\
\hline 22: & -0.9 & $38:-0.8$ & 40: 0.8 & 4: $\quad-0.8$ \\
\hline 16: & -0.7 & 5: -0.7 & 1: $\quad-0.7$ & 18: -0.6 \\
\hline 20: & 0.6 & 17: -0.6 & 36: 0.6 & 34: -0.6 \\
\hline 33: & -0.5 & 19: -0.4 & 3: -0.4 & $24:-0.4$ \\
\hline $12:$ & -0.3 & $21:-0.3$ & $26:-0.3$ & $30:-0.3$ \\
\hline 27: & -0.2 & 2: $\quad 0.2$ & 7: $\quad 0.1$ & $37: \quad 0.0$ \\
\hline 14: & 0.0 & & & \\
\hline $35 /$ & 524 & 423 & 17143.9885 & 50.03570 .010 \\
\hline $15 /$ & 505 & 422 & 9465.9686 & 0.03450 .010 \\
\hline $6 /$ & 625 & 606 & 7721.2599 & 0.03270 .010 \\
\hline 9: & $\begin{array}{llll}3 & 0 & 3 & 2\end{array}$ & 12 & 8521.8259 & 0.02850 .010 \\
\hline 39: & 532 & 431 & 17161.6544 & -0.02810 .010 \\
\hline 23: & 4043 & 313 & 12054.6047 & 0.02620 .010 \\
\hline 13: & 432 & 423 & 9432.0254 & 0.02040 .010 \\
\hline 11: & 330 & 321 & 9408.8109 & -0.01990 .010 \\
\hline 41: & 51444 & 413 & 17354.7895 & -0.01840 .010 \\
\hline 10: & 212 & 101 & 8613.9512 & 0.01800 .010 \\
\hline
\end{tabular}




\section{S4. ${ }^{13} \mathrm{C}_{1} \mathrm{MW}$ Transition Assignments}

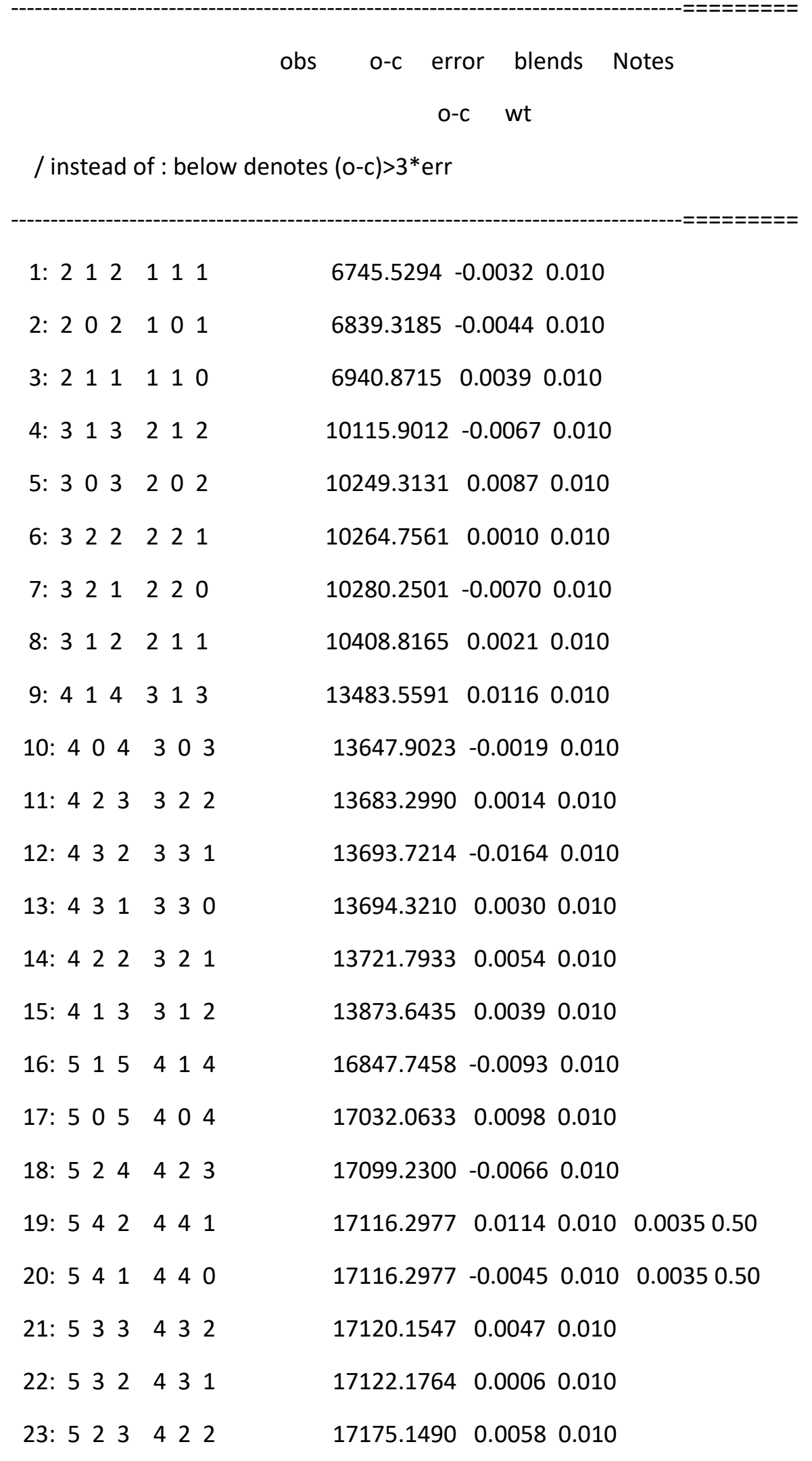


PARAMETERS IN FIT (values truncated):

$\begin{array}{cccc}10000 & \mathrm{~A} / \mathrm{MHz} & 3551.5(1) & 1 \\ 20000 & \mathrm{~B} / \mathrm{MHz} & 1759.636(1) & 2 \\ 30000 & \mathrm{C} / \mathrm{MHz} & 1661.969(1) & 3 \\ 200 & \mathrm{DJ} / \mathrm{kHz} & 0.15(2) & 4 \\ 1100 & \text { DJK } / \mathrm{kHz} & 1.86(6) & 5 \\ 2000 & \mathrm{DK} / \mathrm{kHz} & {[-1.2653205]} & 6 \\ 40100 & \mathrm{~d} 1 / \mathrm{kHz} & {[0.004880261989]} & 7 \\ 50000 & \mathrm{~d} 2 / \mathrm{kHz} & {[0.023032638]} & 8 \\ 1200 & \mathrm{HJK} / \mathrm{Hz} & {[-0.144105325]} & 9 \\ 40200 & \mathrm{~h} 1 / \mathrm{Hz} & {[0.020076864]} & 10 \\ \text { MICROWAVE AVG }= & -0.000072 \mathrm{MHz}, \mathrm{IR} \text { AVG }= & 0.00000 \\ \text { MICROWAVE RMS }= & 0.006968 \mathrm{MHz}, \mathrm{IR} \text { RMS }= & 0.00000 \\ \text { END OF ITERATION } 1 \text { OLD, NEW RMS ERROR= } & 0.69684 & 0.69684 \\ \text { distinct frequency lines in fit: } & 23 \\ \text { distinct parameters of fit: } & 5\end{array}$

upper state lower state overall

$\begin{array}{llllllll}\text { limits of quantum number } & 1: & 2 & 5 & 1 & 4 & 1 & 5\end{array}$

limits of quantum number $2: \begin{array}{llllllll}0 & 4 & 0 & 4 & 0 & 4\end{array}$

limits of quantum number $3: \begin{array}{lllllll}1 & 5 & 0 & 4 & 0 & 5\end{array}$

frequency range: $6745 \quad 17334$

Standard errors are obtained by multiplying the previous errors by: 0.787700

PARAMETERS IN FIT WITH STANDARD ERRORS ON THOSE THAT ARE FITTED:

(values rounded)

$\begin{array}{llll}10000 & \mathrm{~A} / \mathrm{MHz} & 3551.5(1) & 1 \\ 20000 & \mathrm{~B} / \mathrm{MHz} & 1759.637(1) & 2 \\ 30000 & \mathrm{C} / \mathrm{MHz} & 1661.969(1) & 3\end{array}$




$\begin{array}{llll}200 & \mathrm{DJ} / \mathrm{kHz} & 0.15(1) & 4 \\ 1100 & \mathrm{DJK} / \mathrm{kHz} & 1.86(5) & 5 \\ 2000 & \mathrm{DK} / \mathrm{kHz} & {[-1.2653205]} & 6 \\ 40100 & \mathrm{~d} 1 / \mathrm{kHz} & {[0.004880261989]} & 7 \\ 50000 & \mathrm{~d} 2 / \mathrm{kHz} & {[0.023032638]} & 8 \\ 1200 & \mathrm{HJK} / \mathrm{Hz} & {[-0.144105325]} & 9 \\ 40200 & \mathrm{~h} 1 / \mathrm{Hz} & {[0.020076864]} & 10\end{array}$

CORRELATION COEFFICIENTS, C.ij:

A $B \quad$ C $\quad$-DJ $\quad-D J K$

A $\quad 1.0000$

B $\quad 0.2614 \quad 1.0000$

C $\quad-0.2905-0.1364 \quad 1.0000$

-DJ $\quad-0.1530 \quad-0.5766 \quad-0.5813 \quad 1.0000$

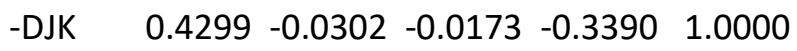

Mean value of $\mid$ C.ij $\mid$, i.ne.j $=0.2816$

Mean value of C.ij, i.ne.j $=-0.1433$

No correlations with absolute value greater than 0.9950

Worst fitted lines (obs-calc/error):

\begin{tabular}{|c|c|c|c|}
\hline 12: -1.6 & 9: 1.2 & 24: -1.1 & 17: 1.0 \\
\hline 16: -0.9 & 5: 0.9 & 7: -0.7 & 4: $\quad-0.7$ \\
\hline 18: -0.7 & 23: 0.6 & 14: 0.5 & 21: 0.5 \\
\hline 2: -0.4 & 3: 0.4 & 15: 0.4 & 19: 0.3 \\
\hline 1: -0.3 & 13: 0.3 & 8: 0.2 & 10: -0.2 \\
\hline 11: 0.1 & 6: 0.1 & 22: 0.1 & 20: 0.0 \\
\hline 12: 432 & 331 & 13693.7214 & $4-0.01640 .010$ \\
\hline 9: 414 & 313 & 13483.5591 & 0.01160 .010 \\
\hline 24: 514 & 413 & 17334.0604 & $4-0.01130 .010$ \\
\hline 17: 505 & 404 & 17032.0633 & $\begin{array}{lll}3 & 0.0098 & 0.010\end{array}$ \\
\hline 16: 515 & 414 & 16847.7458 & $8-0.00930 .010$ \\
\hline
\end{tabular}



5: 3032202
$\begin{array}{lll}10249.3131 & 0.0087 & 0.010\end{array}$
7: 3211220
$10280.2501-0.0070 \quad 0.010$
4: 313212
$10115.9012-0.0067 \quad 0.010$
18: $524 \quad 423$
$17099.2300-0.0066 \quad 0.010$
23: $523 \quad 422$
$17175.1490 \quad 0.0058 \quad 0.010$

/ SPFIT output reformatted with PIFORM 


\section{S5. ${ }^{13} \mathrm{C}_{2} \mathrm{MW}$ Transition Assignments}

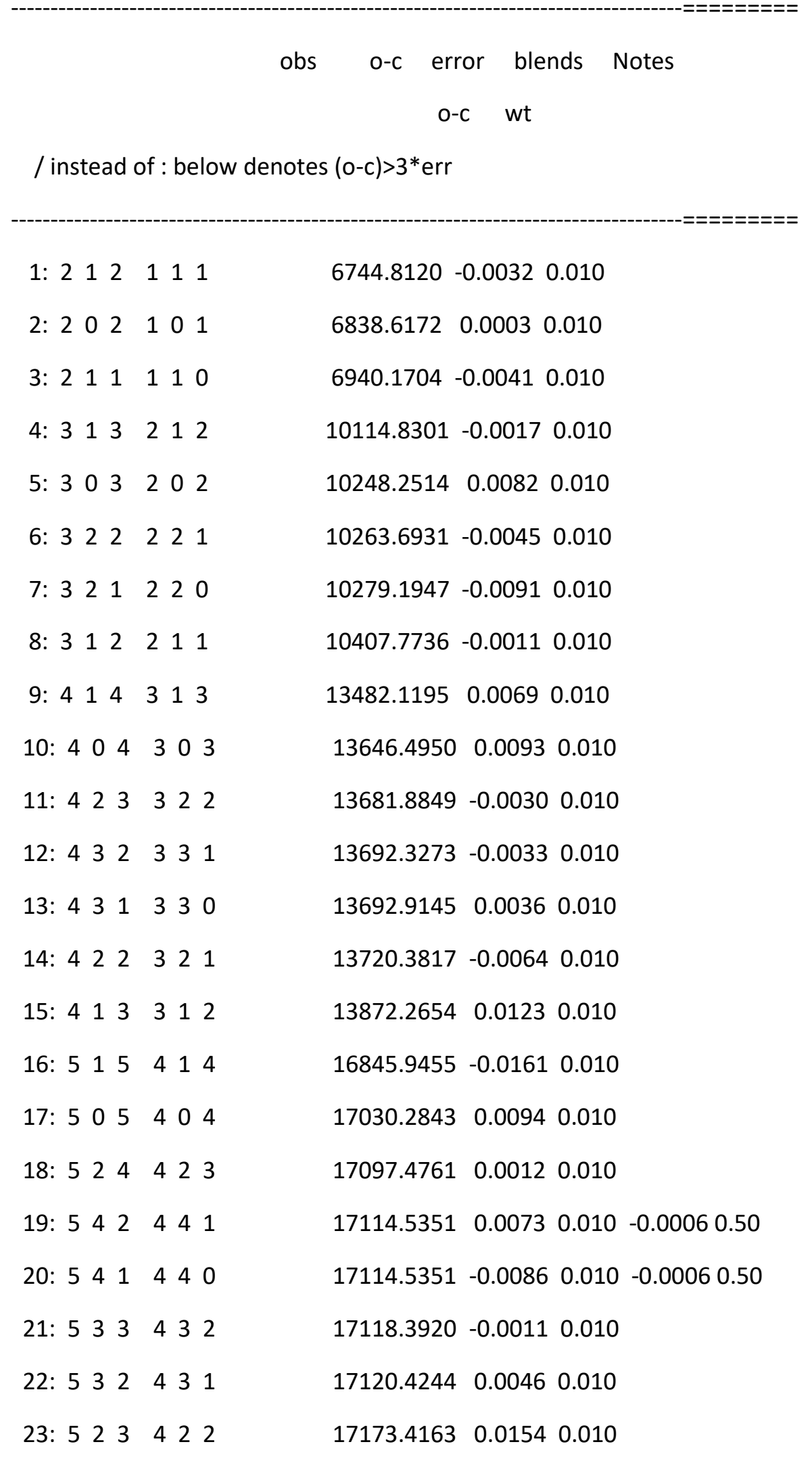


PARAMETERS IN FIT (values truncated):

$\begin{array}{cccc}10000 & \mathrm{~A} / \mathrm{MHz} & 3551.3(1) & 1 \\ 20000 & \mathrm{~B} / \mathrm{MHz} & 1759.466(1) & 2 \\ 30000 & \mathrm{C} / \mathrm{MHz} & 1661.787(1) & 3 \\ 200 & \mathrm{DJ} / \mathrm{kHz} & 0.14(2) & 4 \\ 1100 & \mathrm{DJK} / \mathrm{kHz} & 1.87(6) & 5 \\ 2000 & \mathrm{DK} / \mathrm{kHz} & {[-1.2653205]} & 6 \\ 40100 & \mathrm{~d} 1 / \mathrm{kHz} & {[0.004880261989]} & 7 \\ 50000 & \mathrm{~d} 2 / \mathrm{kHz} & {[0.023032638]} & 8 \\ 1200 & \mathrm{HJK} / \mathrm{Hz} & {[-0.144105325]} & 9 \\ 40200 & \mathrm{~h} 1 / \mathrm{Hz} & {[0.020076864]} & 10 \\ \text { MICROWAVE AVG }= & -0.000161 \mathrm{MHz}, \mathrm{IR} \mathrm{AVG}= & 0.00000 \\ \text { MICROWAVE RMS }= & 0.008323 \mathrm{MHz}, \mathrm{IR} \text { RMS }= & 0.00000 \\ \text { END OF ITERATION } 1 \text { OLD, NEW RMS ERROR= } & 0.83228 & 0.83228 \\ \text { distinct frequency lines in fit: } & 23 \\ \text { distinct parameters of fit: } & 5\end{array}$

upper state lower state overall

$\begin{array}{llllllll}\text { limits of quantum number } & 1: & 2 & 5 & 1 & 4 & 1 & 5\end{array}$

limits of quantum number $2: \begin{array}{llllllll}0 & 4 & 0 & 4 & 0 & 4\end{array}$

limits of quantum number $3: \begin{array}{lllllll}1 & 5 & 0 & 4 & 0 & 5\end{array}$

frequency range: $6744 \quad 17332$

Standard errors are obtained by multiplying the previous errors by: 0.940800

PARAMETERS IN FIT WITH STANDARD ERRORS ON THOSE THAT ARE FITTED:

(values rounded)

$\begin{array}{llll}10000 & \mathrm{~A} / \mathrm{MHz} & 3551.3(1) & 1 \\ 20000 & \mathrm{~B} / \mathrm{MHz} & 1759.467(1) & 2 \\ 30000 & \mathrm{C} / \mathrm{MHz} & 1661.787(1) & 3\end{array}$




$\begin{array}{lcll}200 & \mathrm{DJ} / \mathrm{kHz} & 0.15(2) & 4 \\ 1100 & \mathrm{DJK} / \mathrm{kHz} & 1.87(6) & 5 \\ 2000 & \mathrm{DK} / \mathrm{kHz} & {[-1.2653205]} & 6 \\ 40100 & \mathrm{~d} 1 / \mathrm{kHz} & {[0.004880261989]} & 7 \\ 50000 & \mathrm{~d} 2 / \mathrm{kHz} & {[0.023032638]} & 8 \\ 1200 & \mathrm{HJK} / \mathrm{Hz} & {[-0.144105325]} & 9 \\ 40200 & \mathrm{~h} 1 / \mathrm{Hz} & {[0.020076864]} & 10\end{array}$

CORRELATION COEFFICIENTS, C.ij:

A $\quad$ B $\quad C \quad-D J \quad-D J K$

A $\quad 1.0000$

B $\quad 0.2614 \quad 1.0000$

C $\quad-0.2905-0.1364 \quad 1.0000$

-DJ $\quad-0.1530 \quad-0.5766 \quad-0.5813 \quad 1.0000$

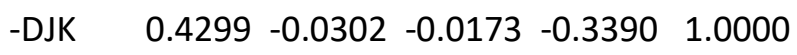

Mean value of $\mid$ C.ij $\mid$, i.ne.j $=0.2816$

Mean value of C.ij, i.ne.j $=-0.1433$

No correlations with absolute value greater than 0.9950

Worst fitted lines (obs-calc/error):

24: $-2.0 \quad 16:-1.6 \quad 23: 1.5 \quad 15: 1.2$

17: $0.9 \quad 10: 0.9 \quad 7: \quad-0.9 \quad 5: 0.8$

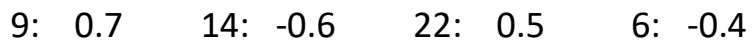

3: $\begin{array}{lllllll}-0.4 & 13: & 0.4 & 12: & -0.3 & 1: & -0.3\end{array}$

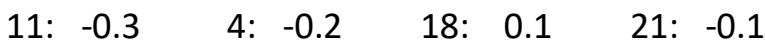

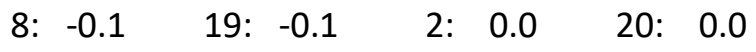

24: $5144413 \quad 17332.3176 \quad-0.02040 .010$

16: $515414 \quad 16845.9455 \quad-0.01610 .010$

23: $52334422 \quad 17173.4163 \quad 0.01540 .010$

15: $4133 \quad 312 \quad 13872.2654 \quad 0.01230 .010$ 


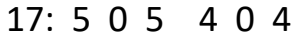

10: 4044303

7: 3212220

5: 303202

9: $414 \quad 313$

14: 4222321
$17030.2843 \quad 0.0094 \quad 0.010$

$13646.4950 \quad 0.0093 \quad 0.010$

$10279.1947-0.00910 .010$

$10248.2514 \quad 0.0082 \quad 0.010$

$13482.1195 \quad 0.00690 .010$

$13720.3817-0.00640 .010$

/ SPFIT output reformatted with PIFORM 


\section{S6. ${ }^{13} C_{3} \mathrm{MW}$ Transition Assignments}

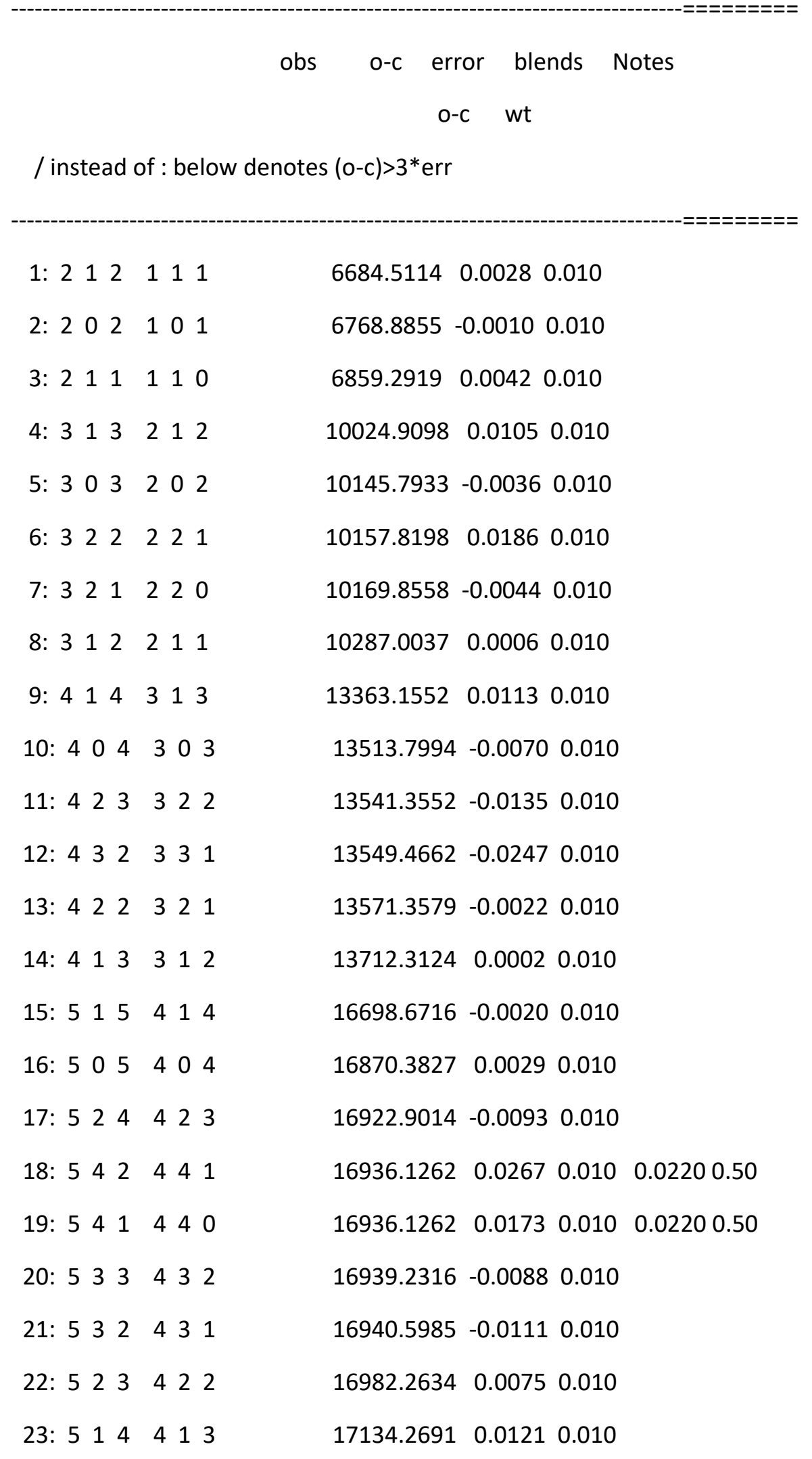


PARAMETERS IN FIT (values truncated):

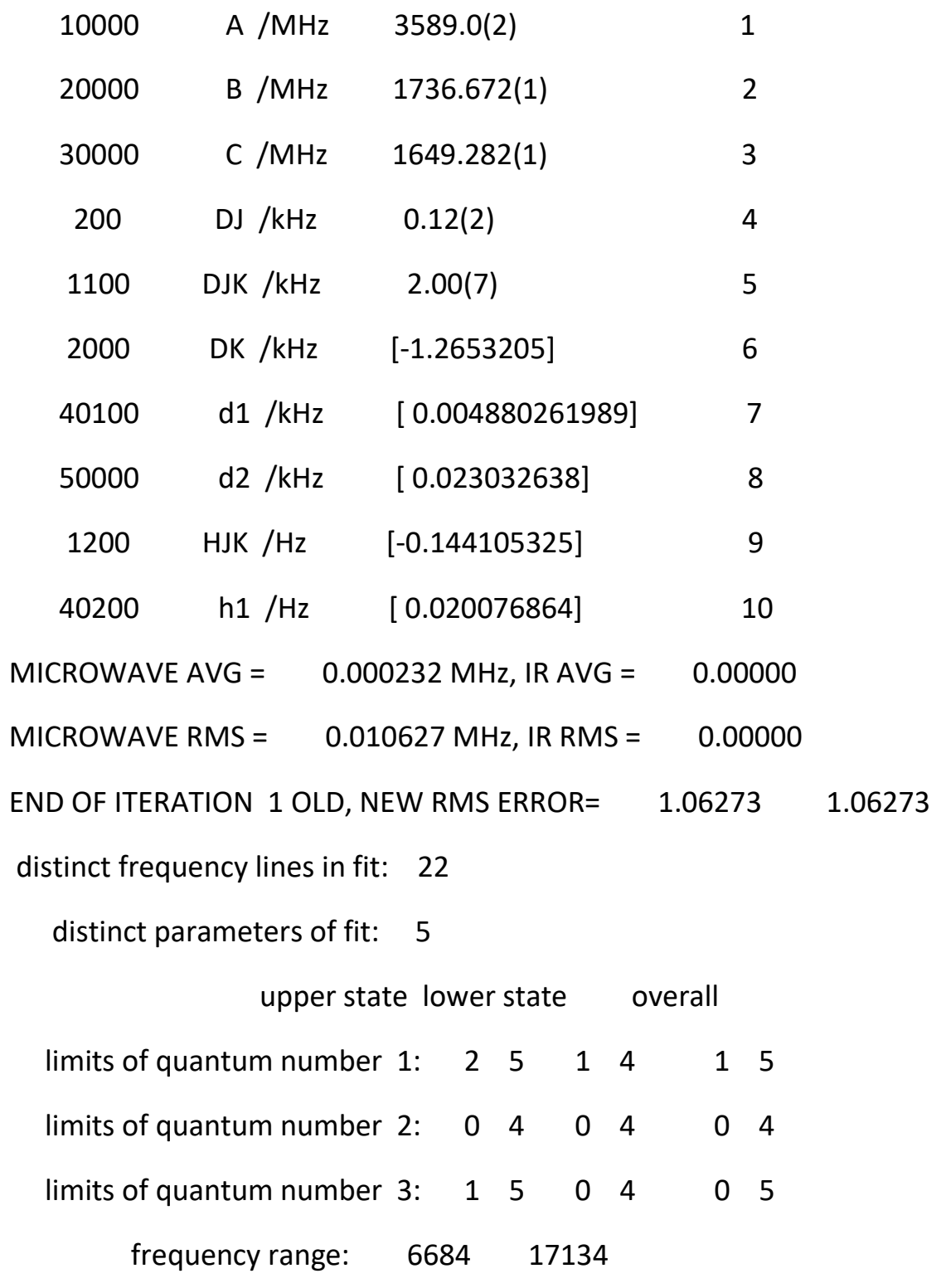

Standard errors are obtained by multiplying the previous errors by: 1.208954

PARAMETERS IN FIT WITH STANDARD ERRORS ON THOSE THAT ARE FITTED:

(values rounded)

$\begin{array}{cccc}10000 & \mathrm{~A} / \mathrm{MHz} & 3589.1(2) & 1 \\ 20000 & \mathrm{~B} / \mathrm{MHz} & 1736.672(1) & 2 \\ 30000 & \mathrm{C} / \mathrm{MHz} & 1649.283(1) & 3 \\ 200 & \mathrm{DJ} / \mathrm{kHz} & 0.13(2) & 4\end{array}$




$\begin{array}{llll}1100 & \text { DJK } / \mathrm{kHz} & 2.01(8) & 5 \\ 2000 & \mathrm{DK} / \mathrm{kHz} & {[-1.2653205]} & 6 \\ 40100 & \mathrm{~d} 1 / \mathrm{kHz} & {[0.004880261989]} & 7 \\ 50000 & \mathrm{~d} 2 / \mathrm{kHz} & {[0.023032638]} & 8 \\ 1200 & \mathrm{HJK} / \mathrm{Hz} & {[-0.144105325]} & 9 \\ 40200 & \mathrm{~h} 1 / \mathrm{Hz} & {[0.020076864]} & 10\end{array}$

Worst fitted constants, with greater than $20 \%$ uncertainty:
$\begin{array}{ccccc}200 & \text { DJ } / \mathrm{kHz} & 0.13(2) & 4 & 20.3\end{array}$

CORRELATION COEFFICIENTS, C.ij:

A $\quad$ B $\quad C \quad-D J \quad-D J K$

A $\quad 1.0000$

B $\quad 0.2344 \quad 1.0000$

C $\quad-0.2540-0.1213 \quad 1.0000$

-DJ $\quad-0.1599-0.5846-0.5891 \quad 1.0000$

$\begin{array}{llllll}\text {-DJK } & 0.4328 & 0.0018 & 0.0137 & -0.3727 & 1.0000\end{array}$

Mean value of $\mid$ C.ij $\mid$, i.ne.j $=0.2764$

Mean value of C.ij, i.ne.j = -0.1399

No correlations with absolute value greater than 0.9950

Worst fitted lines (obs-calc/error):

$\begin{array}{rrrrrrrr}\text { 12: } & -2.5 & 18: & 2.2 & 6: & 1.9 & 11: & -1.3 \\ \text { 23: } & 1.2 & 9: & 1.1 & 21: & -1.1 & 4: & 1.1 \\ \text { 17: } & -0.9 & 20: & -0.9 & 22: & 0.8 & 10: & -0.7 \\ \text { 7: } & -0.4 & 3: & 0.4 & 5: & -0.4 & 16: & 0.3 \\ \text { 1: } & 0.3 & 13: & -0.2 & 15: & -0.2 & 2: & -0.1 \\ \text { 8: } & 0.1 & 14: & 0.0 & 19: & 0.0 & & \end{array}$




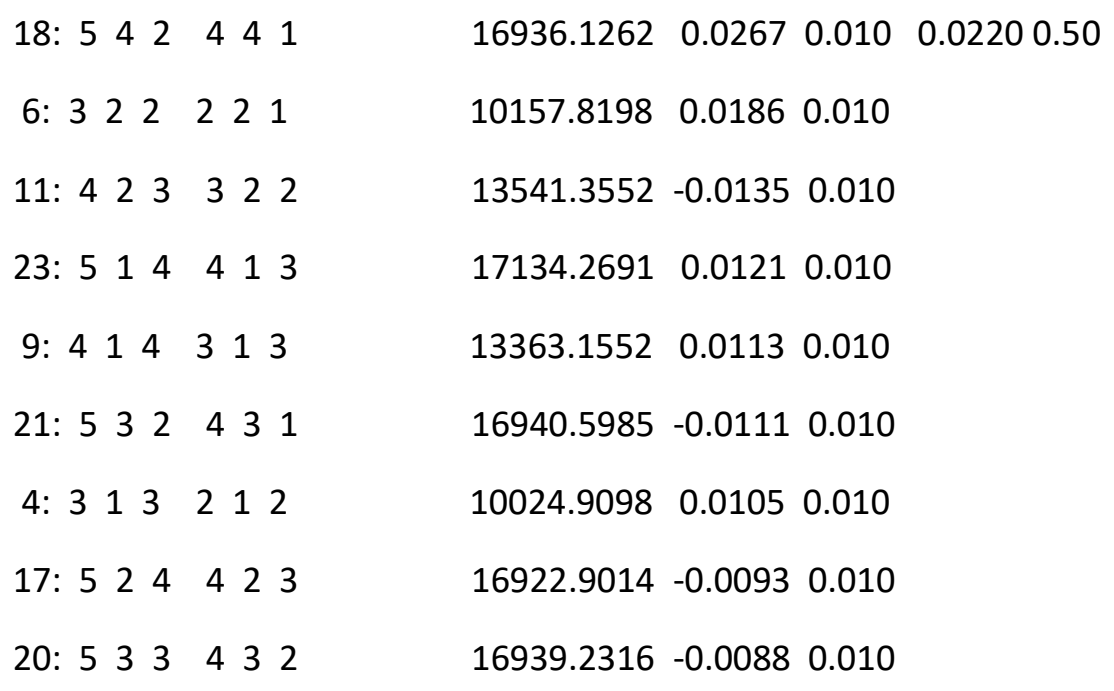

/ SPFIT output reformatted with PIFORM 


\section{S7. ${ }^{13} \mathrm{C}_{4} \mathrm{MW}$ Transition Assignments}

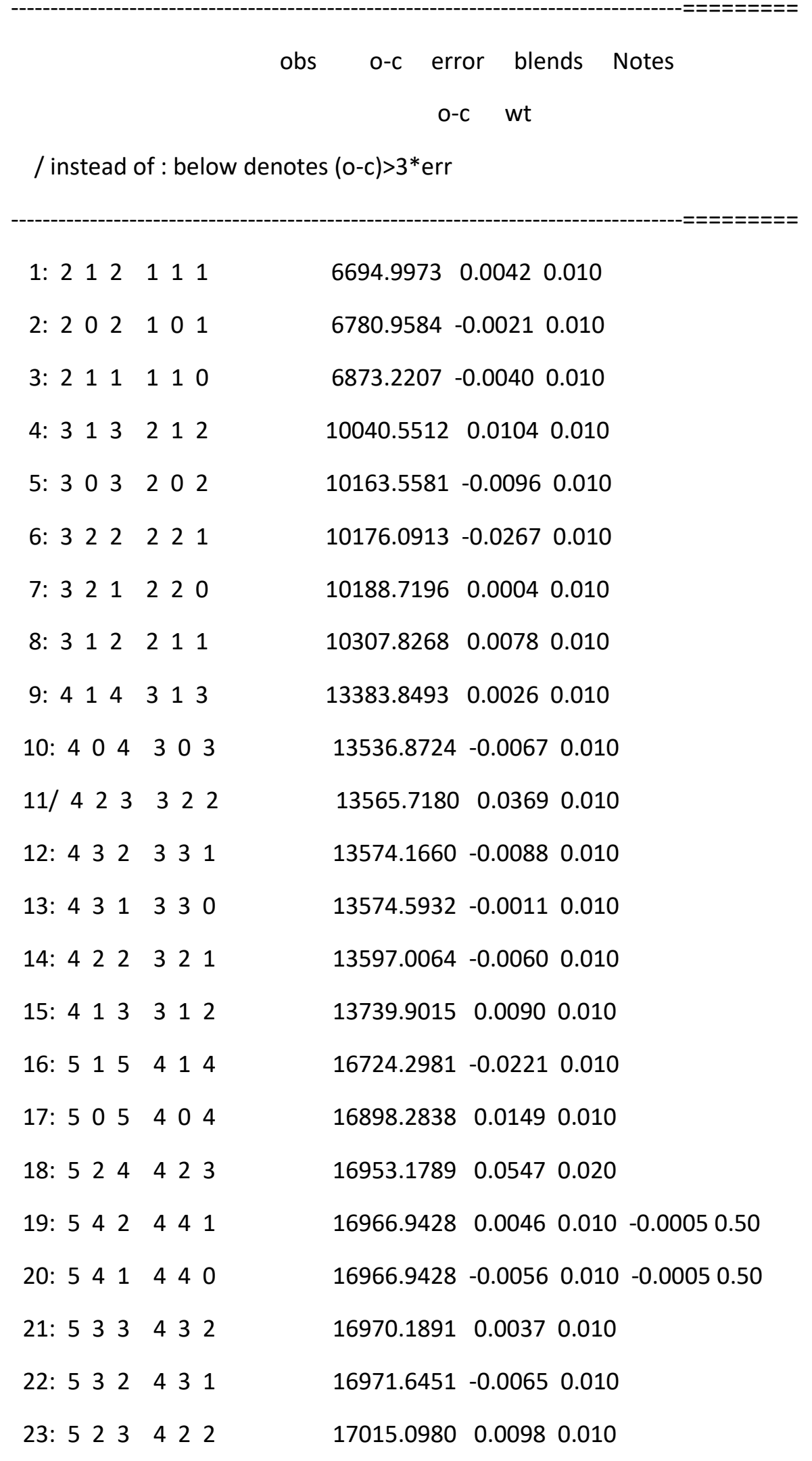


PARAMETERS IN FIT (values truncated):

$\begin{array}{cccc}10000 & \mathrm{~A} / \mathrm{MHz} & 3582.6(2) & 1 \\ 20000 & \mathrm{~B} / \mathrm{MHz} & 1740.588(1) & 2 \\ 30000 & \mathrm{C} / \mathrm{MHz} & 1651.472(1) & 3 \\ 200 & \mathrm{DJ} / \mathrm{kHz} & 0.16(2) & 4 \\ 1100 & \text { DJK } / \mathrm{kHz} & 1.83(6) & 5 \\ 2000 & \mathrm{DK} / \mathrm{kHz} & {[-1.2653205]} & 6 \\ 40100 & \mathrm{~d} 1 / \mathrm{kHz} & {[0.004880261989]} & 7 \\ 50000 & \mathrm{~d} 2 / \mathrm{kHz} & {[0.023032638]} & 8 \\ 1200 & \mathrm{HJK} / \mathrm{Hz} & {[-0.144105325]} & 9 \\ 40200 & \mathrm{~h} 1 / \mathrm{Hz} & {[0.020076864]} & 10 \\ \text { MICROWAVE AVG }= & 0.001651 \mathrm{MHz}, \mathrm{IR} \text { AVG }= & 0.00000 \\ \text { MICROWAVE RMS }= & 0.017439 \mathrm{MHz}, \mathrm{IR} \text { RMS }= & 0.00000 \\ \text { END OF ITERATION } 1 \text { OLD, NEW RMS ERROR= } & 1.43668 & 1.43668 \\ \text { distinct frequency lines in fit: } & 23 \\ \text { distinct parameters of fit: } & 5\end{array}$

upper state lower state overall

$\begin{array}{llllllll}\text { limits of quantum number } & 1: & 2 & 5 & 1 & 4 & 1 & 5\end{array}$

limits of quantum number $2: \begin{array}{llllllll}0 & 4 & 0 & 4 & 0 & 4\end{array}$

limits of quantum number $3: \begin{array}{lllllll}1 & 5 & 0 & 4 & 0 & 5\end{array}$

frequency range: $6694 \quad 17168$

Standard errors are obtained by multiplying the previous errors by: 1.624006

PARAMETERS IN FIT WITH STANDARD ERRORS ON THOSE THAT ARE FITTED:

(values rounded)

$\begin{array}{llll}10000 & \mathrm{~A} / \mathrm{MHz} & 3582.7(3) & 1 \\ 20000 & \mathrm{~B} / \mathrm{MHz} & 1740.588(2) & 2 \\ 30000 & \mathrm{C} / \mathrm{MHz} & 1651.473(2) & 3\end{array}$




$\begin{array}{llll}200 & \mathrm{DJ} / \mathrm{kHz} & 0.17(3) & 4 \\ 1100 & \mathrm{DJK} / \mathrm{kHz} & 1.8(1) & 5 \\ 2000 & \mathrm{DK} / \mathrm{kHz} & {[-1.2653205]} & 6 \\ 40100 & \mathrm{~d} 1 / \mathrm{kHz} & {[0.004880261989]} & 7 \\ 50000 & \mathrm{~d} 2 / \mathrm{kHz} & {[0.023032638]} & 8 \\ 1200 & \mathrm{HJK} / \mathrm{Hz} & {[-0.144105325]} & 9 \\ 40200 & \mathrm{~h} 1 / \mathrm{Hz} & {[0.020076864]} & 10\end{array}$

Worst fitted constants, with greater than $20 \%$ uncertainty:

$\begin{array}{lllll}200 & \text { DJ } / \mathrm{kHz} & 0.17(3) & 4 & 20.9\end{array}$

CORRELATION COEFFICIENTS, C.ij:

$\begin{array}{lrrrrrr} & \text { A } & \text { B } & \text { C } & -D J & -D J K & \\ \text { A } & 1.0000 & & & & \\ \text { B } & 0.2346 & 1.0000 & & & \\ \text { C } & -0.2666 & -0.1308 & 1.0000 & & \\ \text {-DJ } & -0.1482 & -0.5780 & -0.5823 & 1.0000 & \\ \text {-DJK } & 0.4333 & -0.0243 & -0.0123 & -0.3450 & 1.0000\end{array}$

Mean value of $\mid$ C.ij|, i.ne.j = 0.2755

Mean value of C.ij, i.ne.j $=-0.1420$

No correlations with absolute value greater than 0.9950

Worst fitted lines (obs-calc/error):
11: $3.7 \quad 18: \quad 2.7 \quad 6:-2.7 \quad 24:-2.2$
16: $-2.2 \quad 17: 1.5 \quad 4: 1.0 \quad$ 23: 1.0
5: $-1.0 \quad 15: 0.9 \quad 12:-0.9 \quad$ 8: 0.8
10: $-0.7 \quad 22:-0.6 \quad 14:-0.6 \quad$ 1: 0.4

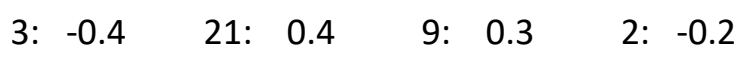
13: $\begin{array}{lllllll}-0.1 & 19: & -0.1 & 7: & 0.0 & 20: & 0.0\end{array}$ 


$\begin{array}{lllllllllll}\text { 11/ } & 4 & 2 & 3 & 3 & 2 & 2 & 13565.7180 & 0.0369 & 0.010 \\ \text { 18: } & 5 & 2 & 4 & 4 & 2 & 3 & 16953.1789 & 0.0547 & 0.020 \\ \text { 6: } & 3 & 2 & 2 & 2 & 2 & 1 & 10176.0913 & -0.0267 & 0.010 \\ \text { 24: } & 5 & 1 & 4 & 4 & 1 & 3 & 17168.4156 & -0.0223 & 0.010 \\ \text { 16: } & 5 & 1 & 5 & 4 & 1 & 4 & 16724.2981 & -0.0221 & 0.010 \\ \text { 17: } & 5 & 0 & 5 & 4 & 0 & 4 & 16898.2838 & 0.0149 & 0.010 \\ \text { 4: } & 3 & 1 & 3 & 2 & 1 & 2 & 10040.5512 & 0.0104 & 0.010 \\ \text { 23: } & 5 & 2 & 3 & 4 & 2 & 2 & 17015.0980 & 0.0098 & 0.010 \\ \text { 5: } 3 & 0 & 3 & 2 & 0 & 2 & 10163.5581 & -0.0096 & 0.010 \\ \text { 15: } & 4 & 1 & 3 & 3 & 1 & 2 & 13739.9015 & 0.0090 & 0.010\end{array}$

/ SPFIT output reformatted with PIFORM 
S8. Output of Structure Evaluation Program for Ring Bond Lengths, Angles, and Dihedral Angles (units are given in $\AA$, degrees, and degrees, respectively) in 1,1-Difluorosilacyclopent-2-ene

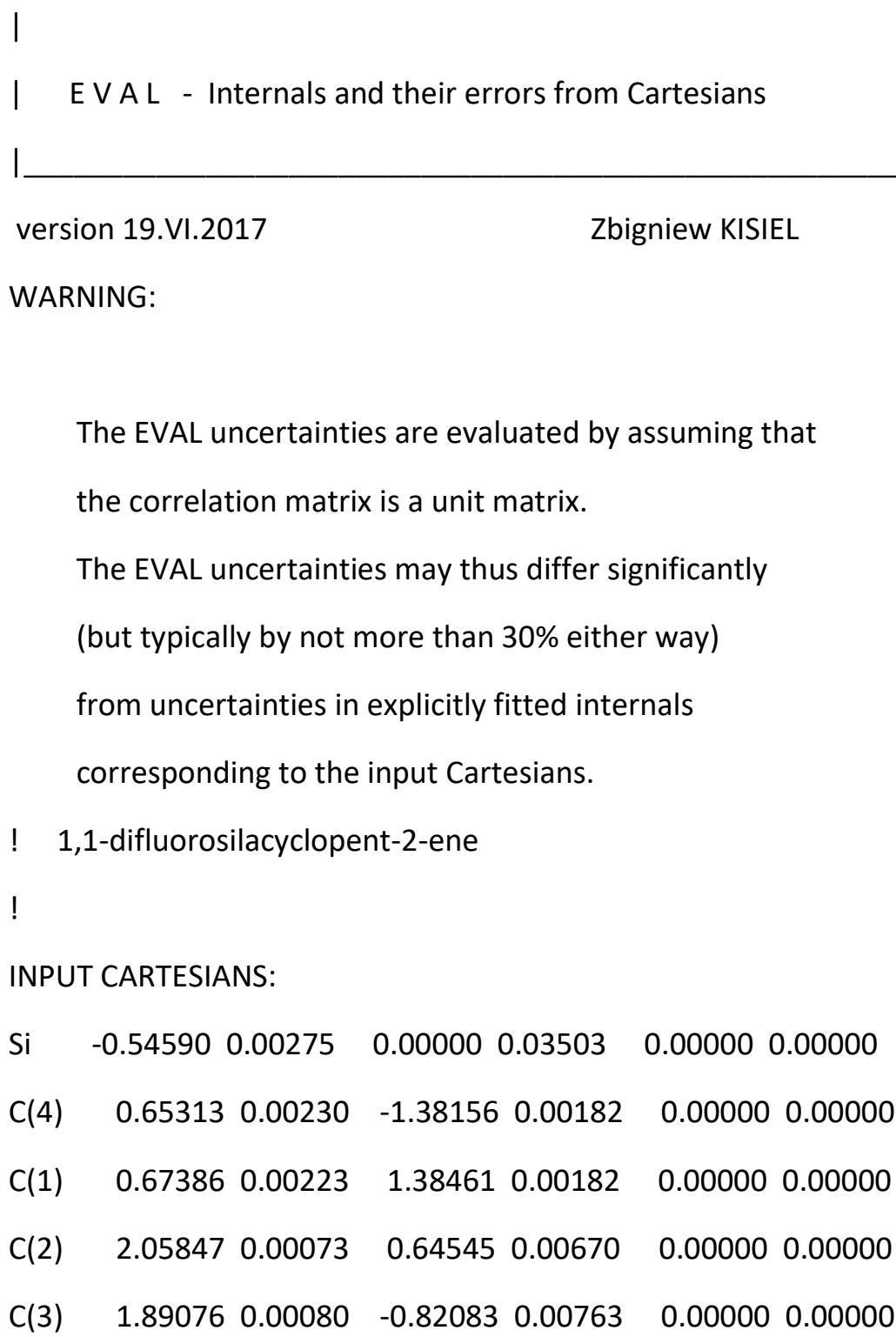

\section{CALCULATED INTERNALS:}

!

! Bond Lengths

!

Si $C(4)=1.82931+-0.02660$ 


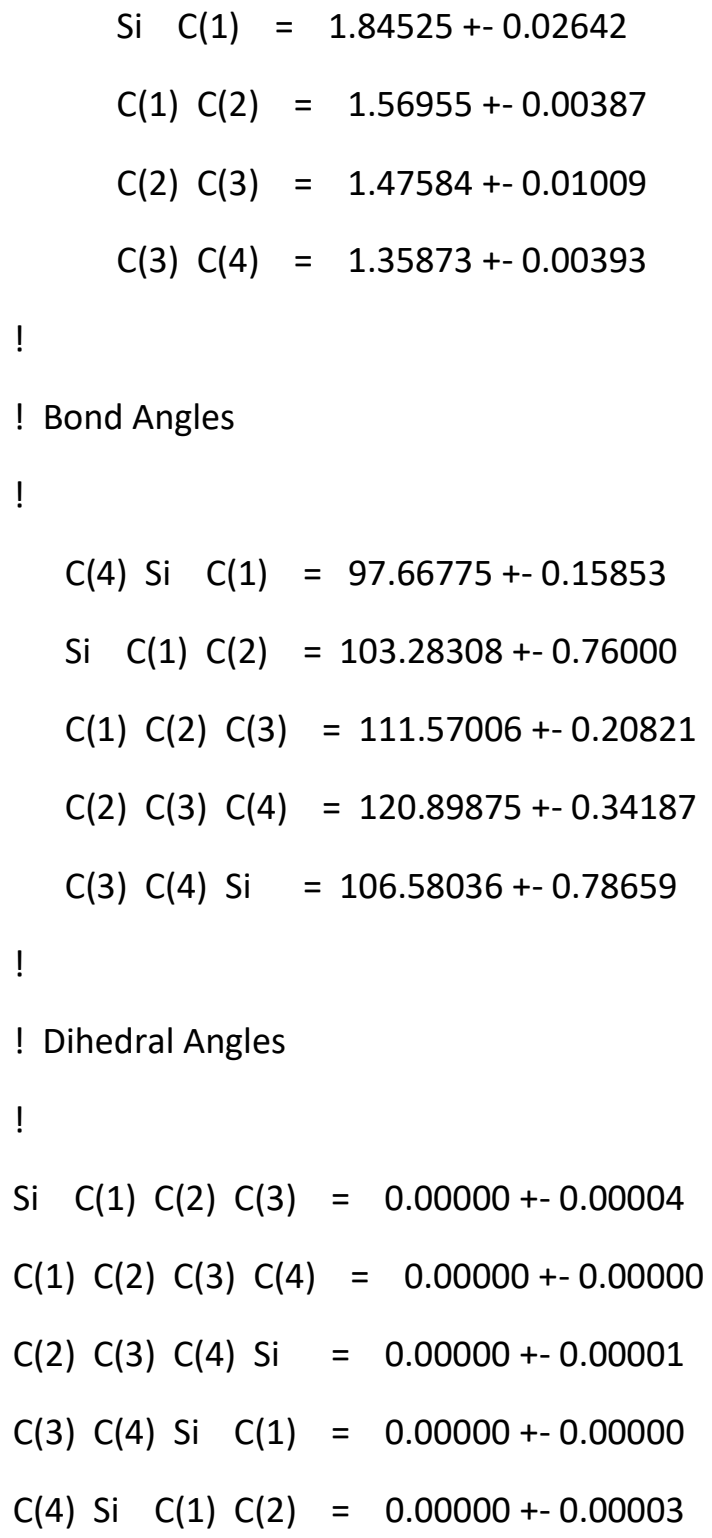


S9. Output of Kraitchman Analysis Program (atom positions are absolute and given in $\AA$ ) in 1,1Difluorosilacyclopent-2-ene

I KRA - SINGLE ISOTOPIC SUBSTITUTION - Various permutations

| of Kraitchman's equations for symmetric/asymmetric tops

।

version 4.IV.2017

Zbigniew KISIEL

1,1-difluorosilacyclopent-2-ene

parent species

Planar calculation will be made from I.a and I.b

$\operatorname{Si}(30)$

The parent species:

\begin{tabular}{lrrl}
$\mathrm{X}, \mathrm{Y}, \mathrm{Z}=$ & 3599.39900000 & 1762.27560000 & 1674.73490000 \\
$\mathrm{eX}, \mathrm{eY}, \mathrm{eZ}=$ & 0.00100000 & 0.00060000 & \multicolumn{1}{l}{0.00050000} \\
$\mathrm{IX}, \mathrm{IY}, \mathrm{IZ}=$ & 140.40649811 & 286.77637533 & 301.76657147 \\
elX,elY,elZ $=$ & 0.00003901 & 0.00009764 & 0.00009009 \\
$\mathrm{PX}, \mathrm{PY}, \mathrm{PZ}=$ & 224.06822435 & 77.69834713 & 62.70815098 \\
& & & \\
Mass $=$ & & &
\end{tabular}

The isotopic species:

$\begin{array}{lrcl}X, Y, Z= & 3599.49100000 & 1758.68500000 & 1671.48600000 \\ \text { eX, eY, eZ }= & 0.00300000 & 0.00100000 & 0.00100000\end{array}$




$\begin{array}{lrcc}\mathrm{IX}, \mathrm{IY}, \mathrm{IZ}= & 140.40290944 & 287.36186918 & 302.35312106 \\ \text { elX,elY,elZ }= & 0.00011702 & 0.00016340 & 0.00018089 \\ \mathrm{PX}, \mathrm{PY}, \mathrm{PZ}= & 224.65604040 & 77.69708066 & 62.70582878\end{array}$

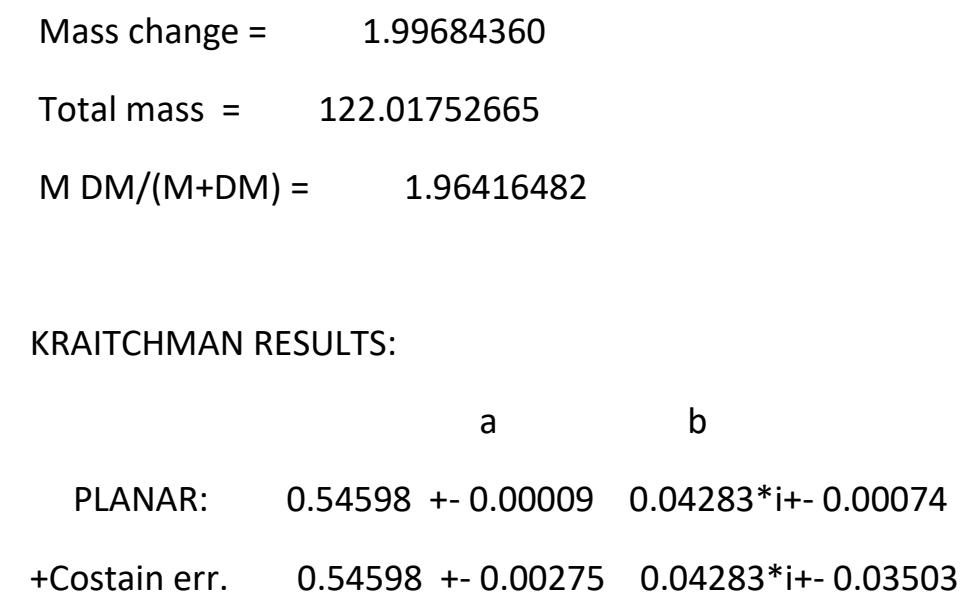




\begin{tabular}{lrrr}
$\mathrm{IX}, \mathrm{IY}, \mathrm{IZ}=$ & 140.40649811 & 286.77637533 & \multicolumn{1}{c}{301.76657147} \\
$\mathrm{eIX}, \mathrm{elY}, \mathrm{eIZ}=$ & 0.00003901 & 0.00009764 & 0.00009009 \\
$\mathrm{PX}, \mathrm{PY}, \mathrm{PZ}=$ & 224.06822435 & 77.69834713 & 62.70815098 \\
& & & \\
Mass $=$ & & &
\end{tabular}

The isotopic species:

$\begin{array}{rrrl}\mathrm{X}, \mathrm{Y}, \mathrm{Z}= & 3600.14500000 & 1760.46300000 & 1673.09200000 \\ \mathrm{eX}, \mathrm{eY}, \mathrm{eZ}= & 0.00700000 & 0.00100000 & 0.00100000 \\ \mathrm{IX}, \mathrm{IY}, \mathrm{IZ}= & 140.37740394 & 287.07164473 & 302.06289248 \\ \text { elX,elY,elZ }= & 0.00027295 & 0.00016307 & 0.00018054 \\ \mathrm{PX}, \mathrm{PY}, \mathrm{PZ}= & 224.37856664 & 77.68432584 & 62.69307810\end{array}$

Mass change $=\quad 0.99956813$

Total mass $=121.02025118$

$\mathrm{M} \mathrm{DM} /(\mathrm{M}+\mathrm{DM})=0.99131219$

KRAITCHMAN RESULTS:

$a \quad b$

$\begin{array}{ccc}\text { PLANAR: } & 0.54582+-0.00018 & 0.17149 * i+-0.00081 \\ \text { Costain err. } & 0.54582+-0.00275 & 0.17149 * i+-0.00878\end{array}$

$\begin{array}{lll}a & b & c\end{array}$

NONPLANAR: $\quad 0.55957+-0.00018 \quad 0.11912 * i+-0.00083 \quad 0.12337 * i+-0.00080$

+Costain err. $\quad 0.55957+-0.002690 .11912 * i+-0.01262 \quad 0.12337 *_{i+-} 0.01218$ 


$$
R=0.53265+-0.00489
$$

$\begin{array}{lrrr}\text { DIX,DIY,DIZ = } & -0.02909417 & 0.29526940 & 0.29632100 \\ \text { DPX,DPY,DPZ = } & 0.31034229 & -0.01402129 & -0.01507289 \\ \text { IXY,IXZ,IYZ = } & -146.36987722 & -161.36007336 & -14.99019614\end{array}$

C1

The parent species:

$\begin{array}{lrcl}\mathrm{X}, \mathrm{Y}, \mathrm{Z}= & 3599.39900000 & 1762.27560000 & 1674.73490000 \\ \mathrm{eX}, \mathrm{eY}, \mathrm{eZ}= & 0.00100000 & 0.00060000 & 0.00050000 \\ \mathrm{IX}, \mathrm{IY}, \mathrm{IZ}= & 140.40649811 & 286.77637533 & 301.76657147 \\ \mathrm{eIX}, \mathrm{eIY}, \mathrm{elZ}= & 0.00003901 & 0.00009764 & 0.00009009 \\ \mathrm{PX}, \mathrm{PY}, \mathrm{PZ}= & 224.06822435 & 77.69834713 & 62.70815098 \\ & & & \\ \text { Mass }= & & & \end{array}$

The isotopic species:

$\begin{array}{lrcl}\mathrm{X}, \mathrm{Y}, \mathrm{Z}= & 3551.50000000 & 1759.63700000 & 1661.96900000 \\ \mathrm{eX}, \mathrm{eY}, \mathrm{eZ}= & 0.10000000 & 0.00100000 & 0.00100000 \\ \mathrm{IX}, \mathrm{IY}, \mathrm{IZ}= & 142.30015737 & 287.20640047 & 304.08449791 \\ \text { elX,elY,elZ }= & 0.00400676 & 0.00016322 & 0.00018297 \\ \mathrm{PX}, \mathrm{PY}, \mathrm{PZ}= & 224.49537050 & 79.58912740 & 62.71102997\end{array}$

Mass change $=\quad 1.00335484$

Total mass $=121.02403789$

$\mathrm{M} \mathrm{DM} /(\mathrm{M}+\mathrm{DM})=\quad 0.99503648$ 
KRAITCHMAN RESULTS:

$a \quad b$

$\begin{array}{ccc}\text { PLANAR: } & 0.65313+-0.00014 & 1.38156+-0.00146 \\ & & \\ \text { Costain err. } & 0.65313+-0.00230 & 1.38156+-0.00182\end{array}$

a $\quad$ b $\quad c$

NONPLANAR: $\quad 0.65094+-0.001531 .38036+-0.000740 .05716+-0.01994$

+Costain err. $\quad 0.65094+-0.002771 .38036+-0.001310 .05716+-0.03296$

$R=1.52721+-0.00208$

DIX,DIY,DIZ = $1.89365926 \quad 0.43002514 \quad 2.31792643$

DPX,DPY,DPZ = $0.42714616 \quad 1.89078028 \quad 0.00287898$

$I X Y, I X Z, I Y Z=-146.36987722 \quad-161.36007336 \quad-14.99019614$

$\mathrm{C} 2$

The parent species:

$\begin{array}{lrcl}\mathrm{X}, \mathrm{Y}, \mathrm{Z}= & 3599.39900000 & 1762.27560000 & 1674.73490000 \\ \mathrm{eX}, \mathrm{eY}, \mathrm{eZ}= & 0.00100000 & 0.00060000 & 0.00050000 \\ \mathrm{IX}, \mathrm{IY}, \mathrm{IZ}= & 140.40649811 & 286.77637533 & 301.76657147 \\ \mathrm{elX}, \mathrm{elY}, \mathrm{elZ}= & 0.00003901 & 0.00009764 & 0.00009009 \\ \mathrm{PX}, \mathrm{PY}, \mathrm{PZ}= & 224.06822435 & 77.69834713 & 62.70815098 \\ & & & \\ \text { Mass }= & & & \end{array}$


The isotopic species:

$\begin{array}{lrrl}\mathrm{X}, \mathrm{Y}, \mathrm{Z}= & 3551.30000000 & 1759.46700000 & 1661.78700000 \\ \mathrm{eX}, \mathrm{eY}, \mathrm{eZ}= & 0.10000000 & 0.00100000 & 0.00100000 \\ \mathrm{IX}, \mathrm{IY}, \mathrm{IZ}= & 142.30817135 & 287.23415040 & 304.11780144 \\ \text { elX,elY,elZ }= & 0.00400721 & 0.00016325 & 0.00018301 \\ \mathrm{PX}, \mathrm{PY}, \mathrm{PZ}= & 224.52189025 & 79.59591119 & 62.71226015\end{array}$

Mass change $=\quad 1.00335484$

Total mass $=121.02403789$

$\mathrm{M} \mathrm{DM} /(\mathrm{M}+\mathrm{DM})=0.99503648$

KRAITCHMAN RESULTS:

a $\quad b$

$\begin{array}{ccc}\text { PLANAR: } & 0.67386+-0.00014 & 1.38461+-0.00146 \\ & & \\ \text { Costain err. } & 0.67386+-0.00223 & 1.38461+-0.00182\end{array}$

$\begin{array}{lll}a & b & c\end{array}$

NONPLANAR: $\quad 0.67083+-0.001491 .38290+-0.000740 .06830+-0.01669$

+Costain err. $\quad 0.67083+-0.002681 .38290+-0.001310 .06830+-0.02759$

$R=1.53853+-0.00206$

$\begin{array}{lrrr}\text { DIX,DIY,DIZ = } & 1.90167323 & 0.45777507 & 2.35122997 \\ \text { DPX,DPY,DPZ = } & 0.45366590 & 1.89756406 & 0.00410917 \\ \text { IXY,IXZ,IYZ = } & -146.36987722 & -161.36007336 & -14.99019614\end{array}$


C3

The parent species:

$\begin{array}{lrrr}\mathrm{X}, \mathrm{Y}, \mathrm{Z}= & 3599.39900000 & 1762.27560000 & 1674.73490000 \\ \mathrm{eX}, \mathrm{eY}, \mathrm{eZ}= & 0.00100000 & 0.00060000 & 0.00050000 \\ \mathrm{IX}, \mathrm{IY}, \mathrm{IZ}= & 140.40649811 & 286.77637533 & 301.76657147 \\ \text { elX,elY,elZ }= & 0.00003901 & 0.00009764 & 0.00009009 \\ \mathrm{PX}, \mathrm{PY}, \mathrm{PZ}= & 224.06822435 & 77.69834713 & 62.70815098 \\ & & & \\ \text { Mass }= & & & \end{array}$

The isotopic species:

$\begin{array}{lrrl}\mathrm{X}, \mathrm{Y}, \mathrm{Z}= & 3589.10000000 & 1736.67200000 & 1649.28300000 \\ \mathrm{eX}, \mathrm{eY}, \mathrm{eZ}= & 0.20000000 & 0.00100000 & 0.00100000 \\ \mathrm{IX}, \mathrm{IY}, \mathrm{IZ}= & 140.80939759 & 291.00429379 & 306.42346335 \\ \text { elX,elY,elZ }= & 0.00784650 & 0.00016756 & 0.00018579 \\ \mathrm{PX}, \mathrm{PY}, \mathrm{PZ}= & 228.30917977 & 78.11428358 & 62.69511402\end{array}$

Mass change $=\quad 1.00335484$

Total mass $=121.02403789$

$M D M /(M+D M)=0.99503648$ 
KRAITCHMAN RESULTS:

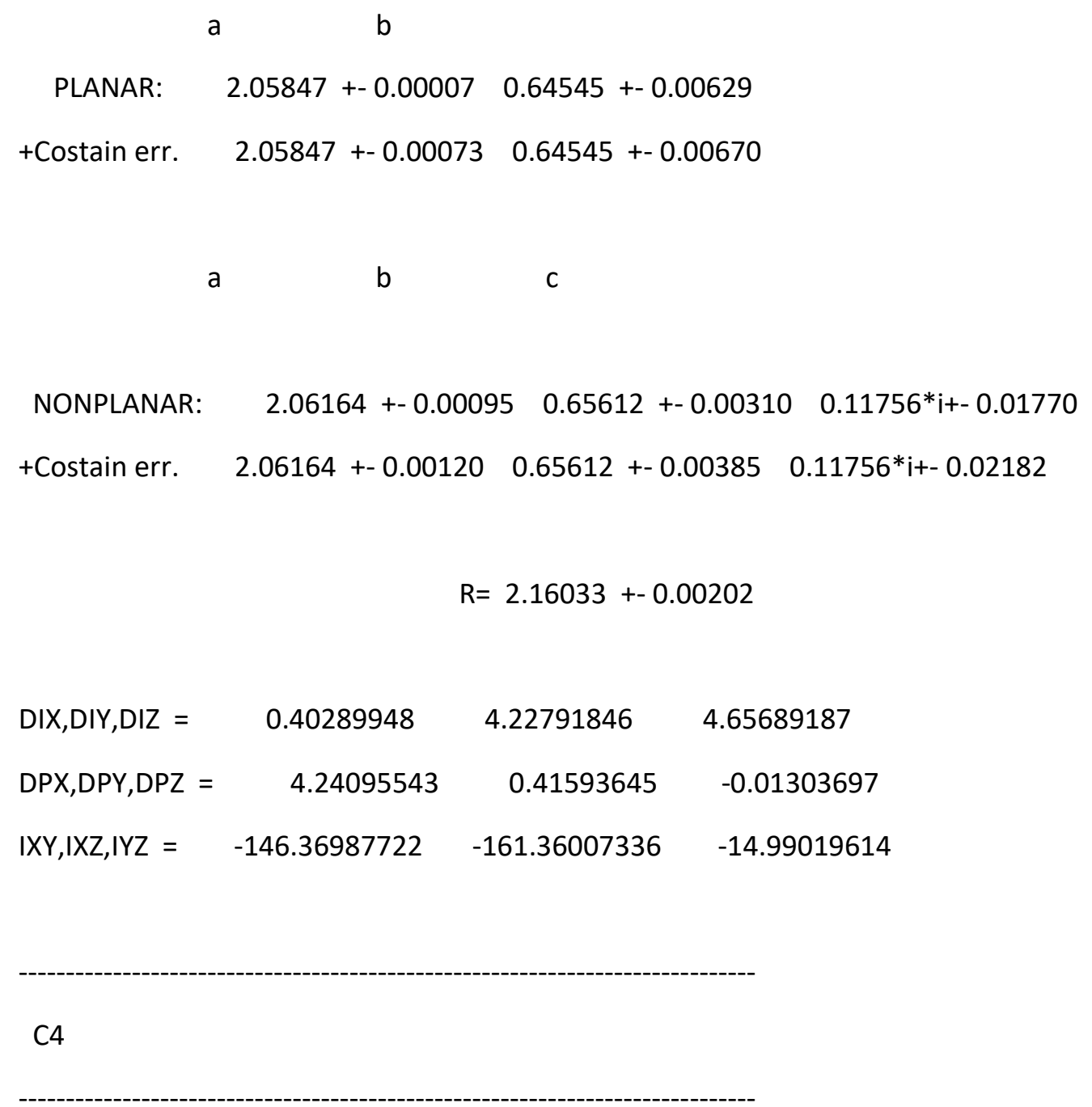

The parent species:

$\begin{array}{lrrr}\mathrm{X}, \mathrm{Y}, \mathrm{Z}= & 3599.39900000 & 1762.27560000 & 1674.73490000 \\ \mathrm{eX}, \mathrm{eY}, \mathrm{eZ}= & 0.00100000 & 0.00060000 & 0.00050000 \\ \mathrm{IX}, \mathrm{IY}, \mathrm{IZ}= & 140.40649811 & 286.77637533 & 301.76657147 \\ \text { elX,elY,elZ }= & 0.00003901 & 0.00009764 & 0.00009009 \\ \mathrm{PX}, \mathrm{PY}, \mathrm{PZ}= & 224.06822435 & 77.69834713 & 62.70815098 \\ & & & \\ \text { Mass }= & 120.02068305 & & \end{array}$


The isotopic species:

$\begin{array}{lrcl}\mathrm{X}, \mathrm{Y}, \mathrm{Z}= & 3582.70000000 & 1740.58800000 & 1651.47300000 \\ \mathrm{eX}, \mathrm{eY}, \mathrm{eZ}= & 0.30000000 & 0.00200000 & 0.00200000 \\ \mathrm{IX}, \mathrm{IY}, \mathrm{IZ}= & 141.06093418 & 290.34958813 & 306.01711860 \\ \mathrm{elX}, \mathrm{elY}, \mathrm{elZ}= & 0.01181184 & 0.00033362 & 0.00037060 \\ \mathrm{PX}, \mathrm{PY}, \mathrm{PZ}= & 227.65288627 & 78.36423233 & 62.69670186\end{array}$

Mass change $=\quad 1.00335484$

Total mass $=121.02403789$

$M D M /(M+D M)=0.99503648$

KRAITCHMAN RESULTS:
a b

$\begin{array}{ccc}\text { PLANAR: } & 1.89076+-0.00012 & 0.82083+-0.00741 \\ & & \\ \text { Costain err. } & 1.89076+-0.00080 & 0.82083+-0.00763\end{array}$

a b c

$\begin{array}{lrlll}\text { NONPLANAR: } & 1.89378+0.00156 & 0.82832+-0.00368 & 0.11083 * i+-0.02861 \\ \text { +Costain err. } & 1.89378+-0.00175 & 0.82832+-0.00410 & 0.11083 * i+-0.03165\end{array}$

$R=2.06404+-0.00286$

DIX,DIY,DIZ = $0.65443607 \quad 3.57321280 \quad 4.25054712$

$\mathrm{DPX}, \mathrm{DPY}, \mathrm{DPZ}=\quad 3.58466192 \quad 0.66588520 \quad-0.01144913$

$I X Y, I X Z, I Y Z=\quad-146.36987722 \quad-161.36007336 \quad-14.99019614$ 